\title{
RENORMALIZATION GROUP APPROACH TO INTERACTING CRUMPLED SURFACES: the hierarchical recursion*
}

\author{
M.Cassandro ${ }^{(*)}$ and P.K.Mitter ${ }^{(+)}$ \\ ${ }^{(* *)}$ Dipartimento di Fisica, I Università di Roma "La Sapienza" \\ P.le A.Moro 2, I-00185 Roma, (Italy) \\ ${ }^{(+)}$Laboratoire de Physique Théorique et Hautes Energies ${ }^{1,2}$ \\ Université Pierre et Marie Curie (Paris 6)
}

PAR-LPTHE 9346

Abstract

We study the scaling limit of a model of a tethered crumpled D-dimensional random surface interacting through an exclusion condition with a fixed impurity in d-dimensional Euclidean space by the methods of Wilson's renormalization group.

In this paper we consider a hierarchical version of the model and we prove rigorously the existence of the scaling limit and convergence to a non-Gaussian fixed point for $1 \leq D<2$ and $\epsilon>0$ sufficiently small, where $\epsilon=D-(2-D) \frac{d}{2}$.

* Work supported by the CNRS and in part by EC Science grant SC*10394 1 URA 280 au CNRS

2 Postal address: LPTHE, Université Paris 6, Boîte 126, 4 Place Jussieu, 75252

Paris Cedex 05, France

e-mail: mitter@lpthe.jussieu.fr cassandro@roma1.infn.it 


\section{Introduction}

There has been much interest recently in the statistical mechanics of tethered surfaces and the associated crumpling transition. [See the contributions in [1] and [2-7]]. The underlying Hamiltonian has stretching and bending terms as well as an Edwards interaction (generalized to surfaces) modelling self-avoidance [See the contributions of D.R.Nelson and others in [1]). In [1 - 7] perturbative renormalisability is assumed and the fixed point in the crumpled phase governing long distance asymptotics is calculated in $\epsilon$-expansion in lowest order. To make further progress in the study of renormalizability Duplantier, in [5], proposed the study of a simpler model where the Edwards interaction corresponding to self-avoidance is replaced by an interaction with a fixed impurity via an exclusion condition. The model is that of the quantum field theory of a fluctuating D-dimensional crumpled surface embedded in d-dimensional Euclidean space corresponding to an Euclidean classical action:

$$
S=\frac{1}{2} \int d^{D} x|\nabla \vec{\phi}(x)|^{2}+\tilde{g}_{0} \int d^{D} x \delta^{(d)}(\vec{\phi}(x))
$$

which we can restrict to a finite volume. Here $\vec{\phi}(x) \varepsilon \Re^{d}$, and represents a point of a D-dimensional surface embedded in $\Re^{d}$. The Gaussian term corresponds to a so called phantom surface whose Haussdorf dimension is identified with $d_{H}=\frac{2 D}{2-D}[1]$. The $\delta$-function interaction in (1.1) forbids this surface from touching a point (in this case the origin) of $R^{d}$. This is highly singular, and thus renormalizability is at stake for the field theory.

However F.David et al [7] have completed the perturbative renormalisation programme to all orders and, for $1 \leq D<2$, found the RG non-Gaussian fixed point in $\epsilon$-expansion. Here $\epsilon=D-(2-D) \frac{d}{2}$. The purpose of this article is to show how the methods of Wilson's renormalization group [8], $(R G)$, can be profitably and rigorously applied to the study of the scaling limit of the Duplantier model. The idea, as usual, is to introduce an ultraviolet (UV) cutoff version of (1.1), and then to remove this cutoff (thus achieving the scaling limit) by thinning out degrees of freedom. through successive RG interations. However, in the present paper, we make one simplification: we replace the cutoff free field by its hierarchical version [Gallavotti [9],[10]]. As is well known the UV cutoff hierarchical free field retains the standard scaling properties and long-distance behaviour but eliminates non-localities 
in the $\mathrm{RG}$ and the resulting $\mathrm{RG}$ transformation is similar to the approximate recursion relation discussed by Wilson [8]. To discuss the "more realistic" theory (without hierarchical approx) it is necessary to take care of the non localities that will arise. Standard techniques to handle this problem are the cluster and Mayer expansions, (see for instance, [10], [11], [12]). This will be the subject of a subsequent paper. But the RG analysis and convergence to a non-Gaussian fixed point is best seen first in the hierarchical framework where the underlying mechanisms are more transparent.

In this paper, starting from an UV cutoff version of (1.1) and in the hierarchical scheme, we will prove the existence of the scaling limit (UV cutoff $\rightarrow \infty$ ), for $1 \leq$ $D<2$ and $\epsilon>0$ sufficiently small. In Section II the cutoff version of the model is presented and in Section III the RG and its hierarchical version. We have gone into some pedagogical detail in Section III for the uninitiated reader. In sections IV and $\mathrm{V}$ we give a rigorous proof of the convergence of the RG iterations to a non-Gaussian fixed point. We estimate the effective potential at every step through convergent expansions. We prove that the sequence of effective potentials converges to a fixed point. The strategy is similar to that of Gawedzki and Kupiainen $[13,14]$ in a different context. The precise statement of our results is given by Theorem 1 at the beginning of section V. Some technical matters are left to the Appendices. 


\section{The UV cutoff model}

We introduce a momentum space cutoff-function $F\left(p^{2}\right)$ where $F>0, C^{\infty}$, monotonic decreasing, $F\left(p^{2}\right) \rightarrow 0$ as $p^{2} \rightarrow \infty$ rapidly and $F(0)=1$. An example of such a cutoff function is

$$
F\left(p^{2}\right)=e^{-p^{2}}
$$

We assume: $\int d^{D} p F\left(p^{2}\right)<\infty$. Our cutoff free field propagator in momentum space is:

$$
C_{\Lambda}(p)=\frac{F\left(p^{2} / \Lambda^{2}\right)}{p^{2}}
$$

where $\Lambda$ is the UV cutoff. We will choose $\Lambda=L^{N}$ (in fixed units), so $\Lambda \rightarrow \infty$ as $N \rightarrow \infty$. Here $L \geq 2$ is an integer. The corresponding cutoff action is:

$$
S_{\Lambda}(\varphi)=\frac{1}{2} \int d^{D} x\left(\nabla \varphi(x), F\left(\frac{-\Delta}{\Lambda^{2}}\right)^{-1} \nabla \varphi(x)\right)+\tilde{g}_{0}(\Lambda) \int d^{D} x \delta_{\Lambda}^{(d)}(\varphi(x))
$$

where $\varphi(x) \epsilon \Re^{d}$ and $(\cdot, \cdot)$ is the inner product in $\Re^{d}$. Here the cutoff dependence of $\tilde{g}_{0}(\Lambda)$ is to be chosen, and $\delta_{\Lambda}^{(d)}$ is an approximating sequence such that $\delta_{\Lambda}^{(d)} \rightarrow \delta^{(d)}$, as $\Lambda \rightarrow \infty$, in the sense of distributions. We will choose:

$$
\delta_{\Lambda}^{(d)}(\varphi)=\left(\frac{\tilde{\lambda}_{0}(\Lambda)}{2 \pi}\right)^{\frac{d}{2}} e^{-\frac{\tilde{\lambda}_{0}(\Lambda)|\varphi|^{2}}{2}}
$$

where $|\cdot|$ is the Euclidean norm in $\Re^{d}, \tilde{\lambda}_{0}>0$ with the property $\tilde{\lambda}_{0}(\Lambda) \rightarrow \infty$ as $\Lambda \rightarrow \infty$. The cutoff dependence will be fixed presently.

From the free field piece of (2.1) we see that the canonical (engineering) dimension of $\varphi$ in mass units, is:

$$
[\varphi]=\frac{D-2}{2}
$$

which means, from $(2.2)$,

$$
\left[\tilde{\lambda}_{0}\right]=2-D
$$

and hence from (2.2) and (2.1) we have:

$$
\left[\tilde{g}_{0}\right]=D-(2-D) \frac{d}{2} \equiv \varepsilon
$$

We can now introduce dimensionless couplings $\lambda_{0}, g_{0}$ via: 


$$
\left.\begin{array}{l}
\tilde{\lambda}_{0}(\Lambda)=\Lambda^{2-D} \lambda_{0} \\
\tilde{g}_{0}(\Lambda)=\Lambda^{\varepsilon} g_{0}
\end{array}\right\}
$$

where $\lambda_{0}, g_{0}$ are cutoff independent and held positive. We shall hold $1 \leq D<2$ and $\varepsilon>0$, so that $\tilde{\lambda}_{0}(\Lambda)$ and $\tilde{g}_{0}(\Lambda) \rightarrow \infty$ as $\Lambda \rightarrow \infty$. (The marginal case $\varepsilon=0$ corresponds to critical internal dimension $D_{\text {crit }}=\frac{2 d}{d+2}$ or critical external dimension $\left.d_{\text {crit }}=\frac{2 D}{2-D}\right)$.

It is convenient to pass to "dimensionless" fields $\Phi$ (unit cutoff) given by:

$$
\varphi(x)=\Lambda^{\frac{D-2}{2}} \Phi(\Lambda x)
$$

Substituting this in (2.1) and making use of (2.6), we get the unit cutoff action:

$$
\begin{aligned}
S_{1}(\Phi) & =\frac{1}{2} \int d^{D} x(\nabla \Phi(x) F(-\Delta) \nabla \Phi(x)) \\
& +\int d^{D} x v_{0}(\Phi(x))
\end{aligned}
$$

where:

$$
v_{0}(\Phi)=g_{0}\left(\frac{\lambda_{0}}{2 \pi}\right)^{\frac{d}{2}} e^{-\frac{\lambda_{0}}{2}|\Phi|^{2}}
$$

To reach the continuum limit $(\Lambda \rightarrow \infty)$ starting from $S_{\Lambda}$ in $(2.1)$, is equivalent to starting from the unit cutoff action (2.8), performing RG iteractions $\ln \Lambda=N$ times and taking $N \rightarrow \infty,[8]$. As we shall see, no unstable directions are encountered, so that no further renormalization is necessary. However to speed up the convergence, and simplify the analysis, we will hold the dimensionless parameter $\lambda_{0}$ at a fixed value $\lambda_{*}$, which will be defined later. 


\section{The R.G. and its hierarchical version}

The partition function corresponding to the unit cutoff action (2.8) is given by:

$$
Z=\int d \mu_{C_{1}}(\Phi) e^{-V_{o}(\Phi)}
$$

where

$$
\left.\begin{array}{c}
\mu_{C_{1}}(\Phi)=\begin{array}{l}
d \\
j=1
\end{array} \mu_{C_{1}}\left(\Phi_{j}\right) \\
V_{0}(\Phi)=\int d^{D} x \quad v_{0}(\Phi(x))
\end{array}\right\}
$$

(see 2.9), and $\mu_{C_{1}}$ is the Gaussian measure of mean 0 and unit cutoff covariance $C_{1}$, whose integral kernel is:

$$
C_{1}(x-y)=\int \frac{d^{D} p}{(2 \pi)^{D}} e^{i p \cdot(x-y)} \frac{F\left(p^{2}\right)}{p^{2}}
$$

(3.1) is well defined, if we restrict ourselves to a finite volume $\vartheta_{N}$, with $\left|\vartheta_{N}\right|=$ $L^{N D}|\vartheta|$. (If the original cutoff action (1.1) is held in fixed volume $\vartheta$, then passing to unit cutoff increases $\vartheta$ to $\vartheta_{N}$ as above).

The sample field $\Phi$ is at least once differentiable, since $\int d^{D} p F\left(p^{2}\right)<\infty$.

To obtain a RG transformation we write

$$
C_{1}=C_{1 / L}+\Gamma
$$

where in momentum space,

$$
\left.\begin{array}{l}
\tilde{C}_{1 / L}(p)=\frac{F\left(L^{2} p^{2}\right)}{p^{2}} \\
\tilde{\Gamma}(p)=\frac{F\left(p^{2}\right)-F\left(L^{2} p^{2}\right)}{p^{2}}
\end{array}\right\}
$$

and, correspondingly, write:

$$
\Phi=\varphi+\zeta
$$

as a sum of independent Gaussian random fields distributed with covariance $C_{1 / L}, \Gamma$ respectively. Correspondingly, the partition function $\mathrm{Z}$ can be written as: 


$$
\begin{aligned}
Z & =\int d \mu_{C_{1 / L}}(\varphi) \int d \mu_{\Gamma}(\zeta) e^{-V_{0}(\varphi+\zeta)} \\
& =\int d \mu_{C_{1}}(\Phi) \int d \mu_{\Gamma}(\zeta) e^{-V_{0}\left(L^{\frac{2-D}{2}} \Phi\left(L^{-1} \cdot\right)+\zeta\right)}
\end{aligned}
$$

The RG transformation then is:

$$
\begin{aligned}
& V_{0}(\Phi) \rightarrow V_{1}(\Phi) \\
& e^{-V_{1}(\Phi)}=\int d \mu_{\Gamma}(\zeta) e^{-V_{0}\left(L^{\frac{2-D}{2}} \Phi\left(L^{-1} \cdot\right)+\zeta\right)}
\end{aligned}
$$

where $\mu_{\Gamma}$ is Gaussian measure with covariance $\Gamma$ (see 3.4). The above transformation is to be iterated $\ln \Lambda=N$ times and $N \rightarrow \infty$, to achieve the continuum limit.

Note that $\Gamma$ has exponential decay in $x$-space, since $\tilde{\Gamma}(p)$ is regular at $p=0$. Even if $V_{0}$ is local, $V_{1}$ will be not. But the RG functional integral, and thus $V_{1}$, can be studied by a high temperature expansion (for this, for other models, see for instance Gallavotti [10], [11], D.C.Brydges [11], and the contributions of Brydges, Gallavotti, Gawedzki- Kupiainen, Feldman et al in [12]).

In this paper we obviate this difficulty by introducing the hierarchical approximation to the cutoff free field, which enforces locality in the RG transformation and keeps scaling properties intact.

To this end we first introduce a sequence of independent Gaussian random vectors $\left\{\zeta_{n}\right\}_{n=0}^{\infty}, \zeta_{n}(x) \varepsilon \Re \Re^{d}$ with covariance

$$
\begin{aligned}
& \left\langle\zeta_{n}^{i}(x) \zeta_{m}^{j}(y)\right\rangle=\delta_{i j} \delta_{n m} \Gamma_{n}(x-y) \\
& \Gamma_{n}(x-y)=\Gamma\left(\frac{x-y}{L^{n}}\right)
\end{aligned}
$$

Then we observe that the unit cutoff free field $\Phi$, distributed according to covariance $C_{1}$, see $(3.3)$, can be written as:

$$
\Phi=\sum_{n=0}^{\infty} L^{n \cdot \frac{(2-D)}{2}} \zeta_{n}
$$

as can be checked by computing its covariance.

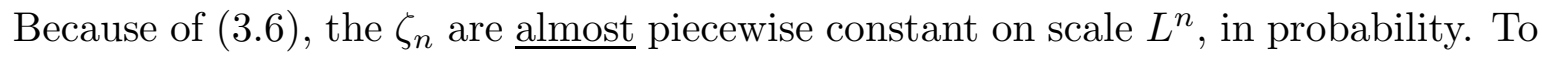
see this we use Tchebycheff's inequality:

$$
\begin{aligned}
P\left\{\left|\zeta_{n}(x)-\zeta_{n}(y)\right|>\delta\right\} & \leq \frac{1}{\delta} E\left(\left|\zeta_{n}(x)-\zeta_{n}(y)\right|^{2}\right) \\
& \leq \frac{|x-y|^{2}}{\delta^{2}} E\left(\left|\nabla \zeta_{n}(\bar{x})\right|^{2}\right)
\end{aligned}
$$


by the mean value theorem, since a sample field $\zeta_{n}$ is at least once differentiable. Now,

$$
\begin{aligned}
E\left(\left|\nabla \zeta_{n}(\bar{x})\right|^{2}\right) & =\frac{d}{L^{2 n}} \int \frac{d^{D} p}{(2 \pi)^{D}}\left(F\left(p^{2}\right)-F\left(L^{2} p^{2}\right)\right) \\
& =\frac{c}{L^{2 n}}, \operatorname{by}(3.6) .
\end{aligned}
$$

Hence for $|x-y| \leq L^{n}$

$$
P\left\{\left|\zeta_{n}(x)-\zeta_{n}(y)\right|>\delta\right\} \leq \frac{c}{\delta^{2}}
$$

which shows that $\zeta_{n}(x)$ is nearly constant on scale $L^{n}$ in probability.

We also record:

$$
\left\langle\left|\zeta_{n}(x)\right|^{2}\right\rangle=d \gamma
$$

where

$$
\gamma=\int \frac{d^{D} p}{(2 \pi)^{D}} \tilde{\Gamma}(p)<\infty
$$

(we will evaluate this later for typical strongly cut off functions $F\left(p^{2}\right)$ ).

Observe also that (3.7), can be written as:

$$
\Phi=\zeta_{0}+L^{\frac{(2-D)}{2}} \bar{\varphi}
$$

where

$$
\bar{\varphi}=\sum_{n=0}^{\infty} L^{n \frac{(2-D)}{2}} \zeta_{n+1}
$$

and it is easy to check, by computing the covariance, that

$$
\bar{\varphi}(x)=\Phi\left(\frac{x}{L}\right)
$$

\section{$\underline{\text { Hierarchical RG }}$}

The hierarchical free field is modelled on (3.7) and the properties of $\zeta_{n}$ explained before. Namely, we replace the Gaussian random vectors $\zeta_{n}$ which are almost constant in probability on scale $L^{n}$ by Gaussian random vectors $\zeta_{\Delta, n}$, which are strictly constant on blocks $\Delta$ of size $L^{n}$. These random vectors are independent for distinct 
blocks of the same size, and also for blocks of different size. The independent Gaussian random vectors have the same covariance $\gamma(3.8)$. Substituting these random vectors in (3.7), gives the hierarchical cutoff free field. Scaling properties are thus preserved.

More precisely, following Gallavotti [9], we introduce a sequence of compatible pavings $\left\{Q_{n}\right\}_{n=0}^{\infty}$, by blocks $\Delta \varepsilon Q_{n}$ of linear size $L^{n}$ of $\Re^{D}$. Here $Q_{n}$ is a refinement of $Q_{n+1}$. To each block $\Delta \varepsilon Q_{n}$ we associate an independent Gaussian random vector $\zeta_{n, \Delta} \varepsilon \Re^{d}$ with covariance:

$$
\left\langle\zeta_{n, \Delta}^{i} \zeta_{m, \Delta^{\prime}}^{j}\right\rangle=\delta_{i j} \delta_{n m} \delta_{\Delta \Delta^{\prime}} \gamma
$$

Let $\Delta_{n}(x)$ be the unique block $\Delta_{n} \in Q_{n}$ with $x \in \Delta_{n}$. The hierarchical cutoff free field is obtained by replacing (3.7) by:

$$
\Phi(x)=\sum_{n=0}^{\infty} L^{n \frac{(2-D)}{2}} \zeta_{\Delta_{n}(x)}
$$

For cutoff $\Lambda$, the fields $\varphi$ live in $\vartheta \subset \Re^{D}$ with $|\vartheta|=L^{M D}$, for $\vartheta$ a hypercube of size $L^{M}$. Hence as observed after (3.3), the unit cutoff field $\Phi$ lives in hypercube of size $L^{N+M}$. Hence (3.12) should be strictly replaced by:

$$
\Phi(x)=\sum_{n=0}^{N+M} L^{n \frac{(2-D)}{2}} \zeta_{\Delta_{n}(x)}
$$

The $\zeta_{\Delta_{n}}$ are piecewise constant, but all scaling properties are preserved. We can write (3.12') as:

$$
\Phi(x)=\zeta_{\Delta_{0}(x)}+L^{\frac{2-D}{2}} \bar{\varphi}_{\Delta_{1}}(x)
$$

where,

$$
\bar{\varphi}_{\Delta_{1}}(x)=\sum_{n=0}^{N+M-1} L^{n \frac{(2-D)}{2)}} \zeta_{\Delta_{n+1}(x)}
$$

$\Delta_{1} \subset \Delta_{2} \subset \Delta_{3} \subset \ldots$. The subscript $\Delta_{1}$ in $\bar{\varphi}_{\Delta_{1}}$ emphasizes that $\bar{\varphi}$ is piecewise constant on blocks $\Delta_{1} \varepsilon Q_{1}$. It is easy to see that $\bar{\varphi}_{\Delta_{1}}(x)=\Phi\left(\frac{x}{L}\right)$, since $x \varepsilon \Delta_{n+1} \Rightarrow$ $\frac{x}{L} \varepsilon \Delta_{n}$.

Our RG transformation (3.5) simplifies considerably for the hierarchical cutoff field. (3.5) reads: 


$$
\left.e^{-V_{1}(\Phi)}=\int \underset{\Delta_{0} \varepsilon Q_{0}}{\Pi} d \mu_{\gamma}\left(\zeta_{\Delta_{0}}\right) e^{-V_{0}\left(\zeta+L^{\frac{2-D}{2}} \bar{\varphi}\right.}\right)
$$

where

$$
d \mu_{\gamma}\left(\zeta_{\Delta_{0}}\right)=(2 \pi \gamma)^{-d / 2} e^{-\frac{\left|\zeta_{\Delta_{0}}\right|^{2}}{2 \gamma}} d^{d} \zeta_{\Delta_{0}}
$$

Since $\Phi$ is piecewise constant over blocks $\Delta_{0} \varepsilon Q_{0}$,

$$
V_{0}(\Phi)=\int d^{D} x \quad v_{0}(\Phi(x))=\sum_{\Delta_{0} \varepsilon Q_{0}} v_{0}\left(\Phi_{\Delta_{0}}\right)
$$

where

$$
\Phi_{\Delta_{0}}=\sum_{n \geq 0} L^{n \frac{(2-D)}{2}} \zeta_{\Delta_{n}}, \quad \Delta_{0} \subset \Delta_{1} \subset \Delta_{2} \subset \ldots
$$

Hence, using (3.15) and (3.13),

$$
\begin{aligned}
e^{-v_{0}\left(\zeta+L \frac{2-D}{2} \bar{\varphi}\right)} & =\underset{\Delta_{0} \varepsilon Q_{0}}{\Pi} e^{-v_{0}\left(\zeta_{\Delta_{0}}+L^{\frac{2-D}{2}} \bar{\varphi}_{\Delta_{1}}\right)} \\
& =\prod_{\Delta_{1} \varepsilon Q_{1}}^{\Pi} \underset{\left(\Delta_{0} \varepsilon Q_{0}\right)}{\Delta_{0} \subset \Delta_{1} e^{-v_{0}\left(\zeta_{\Delta_{0}}+L \frac{2-D}{2} \bar{\varphi}_{\Delta_{1}}\right)}}
\end{aligned}
$$

Plugging this into the RG transformation (3.14), we have

$$
\begin{aligned}
\bar{e}^{V_{1}(\Phi)} & \left.=\underset{\Delta_{1} \varepsilon Q_{1}}{\Pi} \underset{\Delta_{0} \subset \Delta_{0} \varepsilon Q_{0}}{\Pi} \int d \mu_{\gamma}\left(\zeta_{\Delta_{0}}\right) e^{-v_{0}\left(\zeta_{\Delta_{0}}+L^{\frac{2-D}{2}}\right.} \bar{\varphi}_{\Delta_{1}}\right) \\
& \left.=\underset{\Delta_{1} \varepsilon Q_{1}}{\Pi}\left[\int d \mu_{\gamma}\left(\zeta_{\Delta_{0}}\right) e^{-v_{0}\left(\zeta_{\Delta_{0}}+L^{\frac{2-D}{2}}\right.} \bar{\varphi}_{\Delta_{1}}\right)\right]^{L^{D}}
\end{aligned}
$$

since $\sharp\left\{\Delta_{0}\right.$ bloks in $\left.\Delta_{1}\right\}=L^{D}$.

We define the hierarchical RG transformation:

$$
e^{-v_{1}\left(\bar{\varphi}_{\Delta_{1}}\right)}=\left[\int d \mu_{\gamma}\left(\zeta_{\Delta_{0}}\right) e^{-v_{0}\left(\zeta_{\Delta_{0}}+L^{\frac{2-D}{2}} \bar{\varphi}_{\Delta_{1}}\right)}\right]^{L^{D}}
$$

Then from (3.16),

$$
\begin{aligned}
e^{-V_{1}(\Phi)}=\prod_{\Delta_{1} \varepsilon Q_{1}}^{\Pi} e^{-v_{1}\left(\bar{\varphi}_{\Delta_{1}}\right)} & =e^{-\sum_{\Delta_{1} \varepsilon Q_{1}} v_{1}\left(\bar{\varphi}_{\Delta_{1}}\right)}=e^{-\sum_{\Delta_{0} \varepsilon Q_{0}} v_{1}\left(\Phi_{\Delta_{0}}\right)} \\
& =e^{-\int d^{D} x} v_{1}(\Phi(x))
\end{aligned}
$$




\section{Hierarchical RG iterations: the first step}

We begin the study of the sequence of hierarchical RG iterations:

$$
v_{n}(\varphi) \rightarrow v_{n+1}(\varphi), \quad \varphi \varepsilon \Re^{d}
$$

where

$$
v_{n+1}(\varphi)=-L^{D} \ln \left(\left(\mu_{\gamma} * e^{v_{n}}\right)\left(L^{\frac{2-D}{2}} \varphi\right)\right)
$$

where

$$
\begin{gathered}
\left(\mu_{\gamma} * e^{-v_{n}}\right)\left(L^{\frac{2-D}{2}} \varphi\right)=\int d \mu_{\gamma}(\zeta) e^{-v_{n}\left(\zeta+L \frac{2-D}{2} \varphi\right)} \\
d \mu_{\gamma}(\zeta)=(2 \pi \gamma)^{-\frac{d}{2}} e^{-\frac{|\zeta|^{2}}{2 \gamma}} d^{d} \zeta
\end{gathered}
$$

with

$$
v_{0}(\varphi)=g_{0}\left(\frac{\lambda_{0}}{2 \pi}\right)^{\frac{d}{2}} e^{-\frac{\lambda_{0}}{2}|\varphi|^{2}}
$$

Note that $\gamma$, given by (3.8), can be evaluated to be: (use $F\left(p^{2}\right)=e^{-p^{2}}$ as cutoff function)

$$
\gamma=\frac{1}{2^{D / 2}} \cdot \frac{2}{2-D}\left(L^{2-D}-1\right)
$$

and so:

$$
\gamma=O\left(L^{2-D}\right), \text { for } D<2 .
$$

From this, and 4.1 - 4.3, we immediately have analytic continuation in D for

$$
1 \leq D<2
$$

To set the ball rolling, look at the first iteration $v_{0} \rightarrow v_{1}$ in lowest order in $g_{0}$. We have

$$
\begin{aligned}
\left(\mu_{\gamma} * e^{-v_{0}}\right)\left(L^{\frac{2-D}{2}} \varphi\right) & =1-g_{0}\left(\frac{\lambda_{0}}{2 \pi}\right)^{\frac{d}{2}}\left(\mu_{\gamma} * e^{-\lambda_{0}|\cdot|^{2}}\right)\left(L^{\frac{2-D}{2}} \varphi\right)+O\left(g_{0}^{2}\right) \\
& =1-g_{0} L^{-(2-D) \frac{d}{2}}\left(\frac{\lambda_{1}}{2 \pi}\right)^{\frac{d}{2}} e^{-\frac{1}{2} \lambda_{1}|\varphi|^{2}}+O\left(g_{0}^{2}\right)
\end{aligned}
$$


where:

$$
\lambda_{1}=\frac{L^{2-D} \lambda_{0}}{1+\gamma \lambda_{0}}
$$

and we have used the formula for Gaussian integration, for $u>0$,

$$
\left(\mu_{\gamma} * e^{-u|\cdot|^{2}}\right)\left(L^{\frac{2-D}{2}} \varphi\right)=\frac{1}{(1+\gamma u)^{d / 2}} e^{-\frac{1}{2} \frac{L^{2}-D_{u}}{1+\gamma u}|\varphi|^{2}}
$$

Then:

$$
\begin{aligned}
v_{1}(\varphi) & =-L^{D} \ln \left(\mu_{\gamma} * e^{-v_{0}}\left(L^{\frac{2-D}{2}} \varphi\right)\right) \\
& =L^{\varepsilon} g_{0}\left(\frac{\lambda_{1}}{2 \pi}\right)^{\frac{d}{2}} e^{-\frac{1}{2} \lambda_{1}|\varphi|^{2}}+O\left(g_{0}^{2}\right)
\end{aligned}
$$

where:

$$
\varepsilon=D-(2-D) \cdot \frac{d}{2}>0
$$

Notice that we hold $D<2$, hence the map

$$
\lambda_{0} \rightarrow \lambda_{1}=\frac{L^{2-D} \lambda_{0}}{1+\gamma \lambda_{0}}
$$

has a fixed point

$$
\lambda_{*}=\frac{L^{2-D}-1}{\gamma}=2^{-\left(2-\frac{D}{2}\right)}(2-D)<1
$$

To simplify the further analysis, and speed up the convergence, we shall choose $\underline{\text { the starting } \lambda_{0}=\lambda_{*}}$, $\underline{\text { and }}$

$$
v_{*}(\varphi)=\left(\frac{\lambda_{*}}{2 \pi}\right)^{\frac{d}{2}} e^{-\frac{\lambda *}{2}|\varphi|^{2}}
$$

and the starting interaction:

$$
v_{0}(\varphi)=g_{0} v_{*}(\varphi)
$$

$\underline{\text { Iteration } v_{0} \rightarrow v_{1}}$ 


$$
\begin{aligned}
& \beta=2-D, \alpha=(2-D) \frac{d}{2}>0, \quad d \geq 2 . \\
& \varepsilon=D-\alpha>0, \quad 1 \leq D<2\}
\end{aligned}
$$

We shall also write

$$
\langle F\rangle_{\varphi} \equiv\left(\mu_{\gamma} * F\right)\left(L^{\beta / 2} \varphi\right)
$$

Then:

$$
\begin{aligned}
v_{1} & =-L^{D} \ln \left(\left(\mu_{\gamma} * e^{-g_{0} v_{*}}\right)\left(L^{\beta / 2} \varphi\right)\right) \\
& =-L^{D} \ln \left(1+\sum_{n \geq 1} \frac{\left(-g_{0}\right)^{n}}{n !}\left\langle v_{*}^{n}\right\rangle_{\varphi}\right) \\
& =-L^{D} \sum_{k \geq 1} \frac{(-1)^{k-1}}{k}\left(\sum_{n \geq 1} \frac{\left(-g_{0}\right)^{n}}{n !}\left\langle v_{*}^{n}\right\rangle_{\varphi}\right)^{k}
\end{aligned}
$$

whence:

$$
v_{1}(\varphi)=g_{0} L^{D} \sum_{k \geq 1} \sum_{\substack{\ell_{1} \ldots \ell_{k} \\ 1 \leq l_{j}<\infty}}\left(-g_{0}\right)^{|\ell|-1} c_{k}(\ell) \prod_{j=1}^{k}\left\langle v_{*}^{\ell_{j}}\right\rangle_{\varphi}
$$

where:

$$
\begin{gathered}
\ell=\left(\ell_{1}, \ldots, \ell_{k}\right),|\ell|=\sum_{j=1}^{k} \ell_{j} \\
c_{k}(\ell)=\frac{(-1)^{k-1}}{k} \cdot \frac{1}{k} \\
\prod_{j=1}^{k}\left(\ell_{j} !\right)
\end{gathered}
$$

\section{Remark:}

By collecting terms of given power of $g_{0}$, we can write (4.12) as:

$$
v_{1}=L^{D} g_{0} \sum_{n \geq 1} \frac{\left(-g_{0}\right)^{n-1}}{n !}\left\langle v_{*}^{n}\right\rangle_{c o n n}
$$

and this is just the cumulant expansion.

By explicit Gaussian integration, using formula 4.7, together with the property that $\lambda_{*}=\frac{1}{\gamma}\left(L^{2-D}-1\right)$ is the fixed point of the transformation $\lambda \rightarrow \lambda^{\prime}=\frac{L^{\beta} \lambda}{1+\gamma \lambda}$, we obtain, for large $L$, 


$$
\begin{aligned}
\left\langle v_{*}^{\ell}\right\rangle_{\varphi}=L^{-\alpha} v_{*}(\varphi) \frac{1}{\ell^{d / 2}} & \left(\frac{\lambda_{*}}{2 \pi}\right)^{(\ell-1) \frac{d}{2}} \cdot\left(1+\left(1-\frac{1}{\ell}\right) L^{-\beta}\right) . \\
& \cdot \exp \left(-\frac{\lambda_{*}}{2} L^{-\beta}\left(1-\frac{1}{\ell}\right)|\varphi|^{2}\right)
\end{aligned}
$$

where strictly speaking $L^{-\beta}\left(1-\frac{1}{\ell}\right)$ stands for $O\left(L^{-\beta}\left(1-\frac{1}{\ell}\right)\right)$ and this will be understood.

Define:

$$
\begin{aligned}
\tilde{h}_{k}\left(\ell, g_{0}\right) & =\left(-g_{0}\right)^{|\ell|-1} c_{k}(\ell) d_{k}(\ell)\left(\frac{\lambda_{*}}{2 \pi}\right)^{(|\ell|-1) \frac{d}{2}} \\
d_{k}(\ell) & =\prod_{j=1}^{k} \ell_{j}^{-d / 2} \\
f_{k}(\ell) & =\sum_{j=1}^{k}\left(1-\frac{1}{\ell_{j}}\right)
\end{aligned}
$$

with $c_{k}(\ell)$ given in (4.13).

Plugging in (4.14) into (4.12), we get:

$$
v_{1}(\varphi)=L^{\varepsilon} g_{0} v_{*}(\varphi) G_{1}\left(\varphi, g_{0}\right)
$$

where

$$
\begin{gathered}
G_{1}\left(\varphi, g_{0}\right)=\sum_{k \geq 1} L^{-(k-1) \alpha} \sum_{\substack{\ell_{1} \ldots \ell_{k} \\
1 \leq \ell_{j}<\infty}} \tilde{h}_{k}\left(\ell, g_{0}\right)\left(1+L^{-\beta} f_{k}(\ell)\right) . \\
\cdot \exp \left(-\frac{\lambda_{*}}{2}\left[(k-1)+L^{-\beta} f_{k}(\ell)\right]|\varphi|^{2}\right)
\end{gathered}
$$

This is a series of differentiable functions of the variable $\varphi^{2}=|\varphi|^{2} \geq 0$. We have the uniform bound:

$$
\left|G_{1}\right| \leq \sum_{k \geq 1} L^{-(k-1) \alpha} \sum_{\substack{\ell_{1} \ldots \ell_{k} \\ 1 \leq \ell_{j}<\infty}}\left|\tilde{h}_{k}\left(\ell, g_{0}\right)\right|\left(1+L^{-\beta} k\right)
$$

and we claimn that the r.h.s. of (4.18) converges provided

$$
\left|g_{0}\right| \leq\left(\frac{\lambda_{*}}{2 \pi}\right)^{-\frac{d}{2}} \ln \left(1+L^{\alpha}\right)
$$


Thus (4.17) converges uniformly. This shows that $G_{1}$ is a continuous function of $\varphi^{2}$, for sufficiently small $g_{0}$. Later we will see that it is differentiable.

Proof of Claim

To check the uniform convergence, note that

$$
\begin{gathered}
\sum_{\substack{l_{1} \ldots L_{k} \\
1 \leq l_{j}<\infty}}\left|\tilde{h}_{k}\left(\underline{l}, g_{0}\right)\right|\left(1+L^{-\beta} k\right) \\
\leq\left(1+L^{-\beta}\right) \sum_{\substack{l_{1} \ldots l L_{k} \\
1 \leq l_{j}<\infty}} k \cdot \frac{\left(\left|g_{o}\right|\right)^{\left(\sum_{j=1}^{k} l_{j-1}\right)}}{k \cdot \Pi_{j=1}^{k} l_{j} !}\left(\frac{\lambda_{*}}{2 \pi}\right)^{\left(\sum_{j=1}^{k} l j_{-1}\right) \frac{d}{2}} \\
=\frac{\left(1+L^{-\beta}\right)}{\left(\left|g_{0}\right|\left(\frac{\lambda_{*}}{2 \pi}{ }^{d / 2}\right)\right.}\left(\sum_{l \geq 1} \frac{\left(\left|g_{0}\right|\left(\frac{\lambda_{*}}{2 \pi}\right)^{\frac{d}{2}}\right)^{l}}{l !}\right)^{k} \\
=\frac{\left(1+L^{-\beta}\right)}{\left|g_{0}\right|\left(\frac{\lambda_{*}}{2 \pi}\right)^{d / 2}}\left(e^{\left.\left|g_{0}\right|\left(\frac{\lambda_{*}}{2 \pi}\right)^{d / 2}-1\right)^{k}}\right.
\end{gathered}
$$

Putting in the bound $(*)$ in $(4.18)$ we see:

$$
\left|G_{1}\right| \leq\left(1+L^{-\beta}\right)\left[\frac{e^{\left|g_{0}\right|\left(\frac{\lambda_{*}}{2 \pi}\right)^{d / 2}}-1}{\left|g_{0}\right|\left(\frac{\lambda_{*}}{2 \pi}\right)^{d / 2}}\right] \sum_{k \geq 1} \frac{\left(e^{\left|g_{0}\right|\left(\frac{\lambda_{*}}{2 \pi}\right)^{d / 2}}-1\right)^{k-1}}{L^{(k-1) \alpha}}
$$

and the gemetric series on the $r \cdot h \cdot s$ converges provided:

$$
\left|g_{0}\right| \leq \frac{1}{\left(\frac{\lambda_{*}}{2 \pi}\right)^{d / 2}} \cdot \ln \left(1+L^{\alpha}\right)
$$

and the claim is proved.

$\underline{\text { Relevant and Irrelevant terms }}$

We now extract the relevant term which gives an effective coupling $g_{1}$, after one $R G$ step, and a corresponding irrelevant term $I_{1}$, as follows.

Define:

$$
g_{1}=\left.L^{\varepsilon} g_{0} G_{1}\left(\varphi, g_{0}\right)\right|_{\varphi=0}
$$

and

$$
I_{1}\left(\varphi, g_{0}\right) \doteq L^{\varepsilon} g_{0}\left[G_{1}\left(\varphi, g_{0}\right)-G_{1}\left(0, g_{0}\right)\right]
$$

so that we can write the effective potential $v_{1}, 4.16$, as: 


$$
v_{1}(\varphi)=v_{*}(\varphi)\left[g_{1}+I_{1}\left(\varphi, g_{0}\right)\right]
$$

(that $I_{1}$, is irrelevant will be seen presently) From (4.17), and (4.19),

$$
g_{1}=L^{\epsilon} g_{0} \sum_{n \geq 1} a_{n-1} g_{0}^{n-1}
$$

where

$$
a_{n-1}=\left(\frac{\lambda_{*}}{2 \pi}\right)^{(n-1) \frac{d}{2}}(-1)^{n-1} \sum_{k=1}^{n} L^{-(k-1) \alpha} \sum_{\substack{\ell_{1} \ldots \ell_{k} \\|\ell|=n}} c_{k}(\ell) d_{k}(\ell)\left(1+L^{-\beta} f_{k}(\ell)\right)
$$

and (4.22) converges absolutely for: $\left|g_{0}\right|<\left(\frac{\lambda_{*}}{2 \pi}\right)^{-d / 2} \ln \left(1+L^{\alpha}\right)$, as follows from (4.18, $\left.4.18^{\prime}\right)$.

The first few coefficients are:

$$
\begin{aligned}
a_{0} & =1 \\
a_{1} & =-\frac{1}{2}\left(\frac{\lambda_{*}}{4 \pi}\right)^{\frac{d}{2}}\left(1+\frac{L^{-\beta}}{2}-2^{d / 2} L^{-\alpha}\right) . \\
& =-\frac{1}{2}\left(\frac{\lambda_{*}}{4 \pi}\right)^{\frac{d}{2}}\left(1+O\left(L^{-\beta}\right)\right)<0
\end{aligned}
$$

From (4.17) we can write:

$$
g_{1}=L^{\varepsilon} g_{0}\left(1+a_{1} g_{0}\right)+r\left(g_{0}\right)
$$

where

$$
\left|r\left(g_{0}\right)\right| \leq c L^{\varepsilon}\left|g_{0}\right|^{3}
$$

for $g_{0}$ sufficiently small.

Ignoring $r\left(g_{0}\right)$, we derive the approximate fixed point.:

$$
\bar{g}=\frac{L^{\varepsilon}-1}{L^{\varepsilon}\left(-a_{1}\right)}=0(\varepsilon \ln L)>0
$$

Given $\varepsilon>0$, sufficiently small, choose the block size L very large but bounded:

$$
\left(\frac{2}{\varepsilon^{5 / 2}}\right)^{\frac{2}{\beta}}<L<e^{\frac{1}{\varepsilon^{1 / 8}}}
$$

where (see later after 4.31)

$$
\bar{\beta}=\beta-\varepsilon>0
$$


In particular $(4.28) \Rightarrow$

$$
0<\varepsilon<\frac{1}{(\log L)^{8}}
$$

so that

$$
\varepsilon|\ln \varepsilon|<\bar{g}<C \varepsilon^{7 / 8}
$$

Chose the initial $g_{o}$ very close to $\bar{g}$ :

$$
\left|g_{0}-\bar{g}\right|<\frac{1}{4} \quad \varepsilon^{5 / 2}
$$

From $(4.25)$ and

$$
\bar{g}=L^{\varepsilon} \bar{g} \quad\left(1+a_{1} \bar{g}\right)
$$

by taking the difference we can bound (use 4.29)

$$
\left|g_{1}-\bar{g}\right| \leq \frac{1}{2} \varepsilon^{5 / 2}
$$

Hence, from (4.29) and (4.29a), we get

$$
\left|\Delta g_{0}\right|=\left|g_{1}-g_{0}\right| \leq \varepsilon^{5 / 2}
$$

We will show in Section V that (4.30) is not only stable under iteration but contractive:

$$
\left|\Delta g_{n}\right| \leq k_{*}^{n-1}\left|\Delta g_{0}\right|
$$

with $0<k_{*}<1$, and all subsequent effective couplings $g_{n}$ lie within an $\varepsilon^{3 / 2}$ neighbourhood of $\bar{g}$.

We now turn to the irrelevant term $I_{1}(4.20) . I_{1}$ vanishes at $\varphi^{2}=0$, and, by what we have shown for $G_{1}$, is continuous in $\varphi^{2}$. We claim that it is differentiable and satisfies the uniform bound:

$$
\left|\frac{d I_{1}}{d \varphi^{2}}\right| \leq \frac{\bar{g}^{2}}{L^{\bar{\beta} / 2}}
$$

where $\bar{\beta}=\beta-\varepsilon>0\left(\right.$ for $\varepsilon=0, \bar{\beta}_{c}=\beta_{c}=2-D_{c}=\frac{4}{d+2}>0$. Hence for $\varepsilon>0$ sufficiently small $\bar{\beta}>0$ by continuity)

To see this take the derivative of (4.17) term by term and upper bound. Note that the $k=l=1$ term in 4.17 gives 1 and so does not contribute to $I_{1}$. Also, after 
taking the derivative, the $\varphi^{2}$-dependent terms (exponentials with negative exponents) can be bounded by 1 . Hence

$$
\begin{aligned}
& \left|\frac{d I_{1}}{d \varphi^{2}}\right| \leq L^{\varepsilon}\left|g_{0}\right| \frac{\lambda_{*}}{2} L^{-\beta}\left(1+L^{-\beta}\right) . \\
& \cdot\left\{\sum_{l \geq 2}\left|\tilde{h}_{1}\left(l, g_{0}\right)\right|+\left(1+L^{-\beta}\right) \sum_{k \geq 2} L^{-(k-2)} \cdot \sum_{\substack{l_{1} \ldots l_{k} \\
l_{j} \geq 1}} k^{2}\left|\tilde{h}_{k}\left(l, g_{0}\right)\right|\right\}
\end{aligned}
$$

If we now plug in the expression $(4.15,4.13)$ for $\tilde{h}_{k}$, we can easily verify that the series in braces \{\} converge for $\left|g_{0}\right|<\left(\frac{\lambda_{*}}{2 \pi}\right)^{-d / 2} \ln \left(1+L^{\alpha}\right)$ and \{\} is $0\left(g_{0}\right)$. We restrict our selves to $\frac{1}{4} \varepsilon^{5 / 2}$ neigbourhood of $\bar{g}$ as in (4.29). Then we have:

$$
\left|\frac{d I_{1}}{d \varphi^{2}}\right| \leq \frac{C \bar{g}^{2}}{L^{\bar{\beta}}}
$$

We use up a factor $\frac{1}{L^{\beta / 2}}$ to bound the constant by 1 . We then get (4.31). Of course we can get a much stronger bound (as far as the field dependence is concerned), but we will not need it. In fact we shall replace (4.31) by a weaker bound:

$$
\left|\frac{d I_{1}}{d \varphi^{2}}\right| \leq \frac{\bar{g}^{2}}{L^{\bar{\beta} / 2}} e^{\frac{\lambda *}{2} L^{-\beta}|\varphi|^{2}}
$$

and by integrating this from 0 to $\varphi^{2}$, with $I_{1}(0)=0$ we get:

$$
\left|I_{1}\right| \leq \frac{\bar{g}^{2}}{L^{\bar{\beta} / 2}} \varphi^{2} e^{\frac{\lambda_{*}}{2} L^{-\beta}|\varphi|^{2}}
$$

The growth in $\varphi^{2}$ is harmless since, from (4.21), $I_{1}$ is always multiplied by

$$
v_{*}=\left(\frac{\lambda_{*}}{2 \pi}\right)^{d / 2} e^{-\frac{\lambda_{*}}{2}|\varphi|^{2}}
$$

and

$$
\sup _{\varphi}\left|v_{*} I_{1}\right| \leq \frac{\bar{g}^{2}}{L^{\bar{\beta} / 2}}
$$

We will see in Section V that the bound (4.32) is stable under iteration.

We summarize what we have obtained after one iteration in the following Proposition

\section{$\underline{\text { Proposition } 1}$}

Let $\varepsilon>0$ be sufficiently small (4.28) and $\bar{g}$ be defined by (4.27). Hold $g_{0}$ so that

$$
\left|g_{0}-\bar{g}\right| \leq \frac{1}{4} \varepsilon^{5 / 2}
$$


Then after 1 RG iteration

$$
v_{0}=g_{0} v_{*} \rightarrow v_{*}\left(g_{1}+I_{1}\right)
$$

where $I_{1}(0)=0 . I_{1}(\varphi)$ is $C^{1}$ in $\varphi^{2}$ and the following bounds hold:

$$
\begin{aligned}
& \left|\Delta g_{0}\right|=\left|g_{1}-g_{0}\right| \leq \varepsilon^{5 / 2} \\
& \left|\frac{d I_{1}}{d \varphi^{2}}\right| \leq \frac{\bar{g}^{2}}{L^{\bar{\beta} / 2}} e^{\frac{\lambda *}{2} L^{-\beta}|\varphi|^{2}}
\end{aligned}
$$

where $\bar{\beta}=\beta-\varepsilon>0$. 


\section{Higher iterations and Convergence to non Gaussian fixed point}

Let us write, in analogy to what we have obtained after one iteration, the effective potential $V_{n}$ after the $n t h R G$ iteration in the form:

$$
v_{n}(\varphi)=v_{*}(\varphi)\left[g_{n}+I_{n}(\varphi)\right]
$$

where $I_{n}(0)=0, \underline{I_{n} \text { being the irrelevant term. }}$. Recall, $v_{*}=\left(\frac{\lambda_{*}}{2 \pi}\right)^{d / 2} e^{-\frac{\lambda_{*}}{2}|\varphi|^{2}}$ and define the uniform norm of $v_{n}$ :

$$
\left\|v_{n}\right\|=\left(\frac{\lambda_{*}}{2 \pi}\right)^{d / 2}\left(\left|g_{n}\right|+\left\|I_{n}\right\|_{v_{*}}\right)
$$

where

$$
\left\|I_{n}\right\|_{v_{*}}=\sup _{\varphi}\left|e^{\frac{-\lambda_{*}}{2}|\varphi|^{2}} I_{n}(\varphi)\right|
$$

In this section we will prove our main Theorem:

Theorem 1 (Convergence to non-Gaussian fixed point)

As $n \rightarrow \infty, v_{n} \rightarrow v_{\infty}$ in the uniform norms $\|\cdot\|$ where

$$
\left\|v_{\infty}\right\|=\left(\frac{\lambda_{*}}{2 \pi}\right)^{d / 2}\left(\left|g_{\infty}\right|+\left\|I_{\infty}\right\|_{v_{*}}\right)
$$

and

$$
\begin{aligned}
\left|g_{\infty}-\bar{g}\right| & \leq \varepsilon^{3 / 2} \\
\left\|I_{\infty}\right\|_{v_{*}} & \leq \frac{4 \bar{g}^{2}}{L^{\bar{\beta} / 2}}
\end{aligned}
$$

provided $\varepsilon>0$ is sufficiently small and the block size L sufficiently large (the precise condition is (4.28) of Section IV) Here,

$$
\bar{g}=\frac{L^{\varepsilon}-1}{L^{\varepsilon}\left(-a_{1}\right)}=0(\varepsilon \ln L)>0
$$

is the approximate fixed point of the first iteration, and

$$
\bar{\beta}=\beta-\varepsilon=(2-D)-\varepsilon>0
$$

(infact for $\varepsilon=0, \bar{\beta}_{c}=2-D_{c}=2-\frac{2 d}{d+2}=\frac{4}{d+2}>0$ and hence, by continuity it follows that $\bar{\beta}>0$ for $\varepsilon>0$ very small). $\underline{\text { Note that } g_{\infty}>0 \text { because of }(5.3) \text { and (4.28a). }}$ 
Theorem 1 thus states convergence of the sequence of effective potentials to a nonGaussian fixed point close to the approximate fixed point of the first iteration.

In order to prove Theorem 1 we shall bound the difference of successive iterations:

$$
\Delta v_{n}=v_{n+1}-v_{n}=v_{*}\left(\Delta g_{n}+\Delta I_{n}\right)
$$

(our strategy is similar to that of Gawedzki and Kupiainen in $[13,14]$, in a different context)

To this end we shall make an inductive hypothesis (verified for $n=1$ ) for the first n-steps of RG iteration. As in section IV, (4.28) choose the block size L very large but bounded and $\varepsilon>0$ very small such that:

$$
\frac{1}{\left(\frac{1}{2} L^{\bar{\beta} / 2}\right)^{2 / 5}}<\varepsilon<\frac{1}{(\ln L)^{8}}
$$

This is easy to fulfill, as the reader can check. Since $\bar{g}=0(\varepsilon \ln L)$, the righthand inequality assures us that,

$$
\bar{g}^{2}<C \varepsilon^{7 / 4}=\varepsilon^{3 / 2}\left(C \varepsilon^{1 / 4}\right)
$$

which is very small. Define:

$$
k_{*}=1-\varepsilon \ln L+10 \varepsilon^{3 / 2}
$$

Note that,

$$
0<k_{*}<1
$$

Define also:

$$
\begin{gathered}
\delta(\varepsilon)=\varepsilon^{5 / 2} \\
\delta_{*}(\varepsilon)=\varepsilon^{3 / 2}
\end{gathered}
$$

and hold the initial coupling $g_{0}$ as in Section IV:

$$
\left|g_{0}-\bar{g}\right|<\frac{1}{4} \delta(\varepsilon)
$$

Define:

$$
\begin{aligned}
\Delta g_{l} & =g_{l+1}-g_{l} \\
\Delta I_{l} & =I_{l+1}-I_{l}
\end{aligned}
$$


* Inductive hypothesis: For the first n-steps of the RG iteration, the effective potential $v_{1}, v_{2}, \ldots, v_{n}$ satisfy the following, Property $H_{n}$ :

For $l=0,1,2, \ldots, n-1$

$$
\begin{aligned}
& \text { (i) }\left|\Delta g_{l}\right| \leq k_{*}^{l} \delta(\varepsilon) \\
& \text { (ii) }\left|\frac{\mathrm{d}}{d \varphi^{2}} \Delta I_{l}\right| \leq c^{(l)} e^{\frac{\lambda_{*}}{2} L^{-\beta}|\varphi|^{2}}
\end{aligned}
$$

where:

$$
c^{(0)}=\frac{\bar{g}^{2}}{L^{\bar{\beta} / 2}}
$$

and for $l \geq 1$ :

$$
c^{(l)}=\frac{\bar{g}}{L^{\bar{\beta} / 2}} k_{*}^{l-1} \delta(\varepsilon)+\frac{\bar{g}^{2}}{L^{\bar{\beta} / 2}}\left(\frac{1}{L^{\bar{\beta} / 2}}\right)^{l}
$$

Note that for $n=1$, the inductive hypothesis is satisfied (Proposition 1 of Section IV).

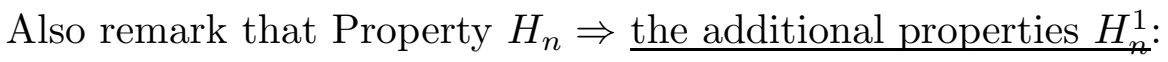

Property $H_{n}^{1}$

$$
\begin{gathered}
\text { (ia) }\left|g_{n}-\bar{g}\right| \leq \varepsilon^{3 / 2}=\delta_{*}(\varepsilon) \\
\text { (iia) }\left|\frac{d}{d \varphi^{2}} I_{n}\right| \leq \frac{4 \bar{g}^{2}}{L^{\bar{\beta} / 2}} e^{\frac{\lambda_{*}}{2} L^{-\beta}}|\varphi|^{2} \\
\text { (iib) }\left|\Delta I_{n-1}\right| \leq c^{(n-1)}|\varphi|^{2} e^{\frac{\lambda_{*}}{2} L^{-\beta}}|\varphi|^{2} \\
\text { (iic) }\left|I_{n}\right| \leq \frac{4 \bar{g}^{2}}{L^{\bar{\beta} / 2}}|\varphi|^{2} e^{\frac{\lambda_{*}}{2} L^{-\beta}|\varphi|^{2}}
\end{gathered}
$$

Proof. Start from $H_{n}$

(i) $\Rightarrow$ (ia), since

$$
\begin{aligned}
& \left|g_{n}-\bar{g}\right| \leq \sum_{l=0}^{n-1}\left|\Delta g_{l}\right|+\left|g_{0}-\bar{g}\right| \leq\left(\sum_{l=0}^{n-1} k_{*}^{l}\right) \delta(\varepsilon)+\frac{1}{2} \delta(\varepsilon) \\
& \leq \frac{1}{1-k_{*}} \varepsilon^{5 / 2}+\frac{1}{2} \varepsilon^{5 / 2} \\
& \leq \varepsilon^{3 / 2}
\end{aligned}
$$

Next, (ii) $\Rightarrow$ (ii a), since

$$
\left|\frac{d}{d \varphi} I_{n}\right| \leq \sum_{l=0}^{n-1}\left|\frac{d}{d \varphi^{2}} \Delta I_{l}\right| \leq\left(\sum_{l-0}^{n-1} c^{(l)}\right) e^{\frac{\lambda_{*}}{2} L^{-\beta}|\varphi|^{2}}
$$

and

$$
\sum_{l=0}^{n-1} c^{(l)} \leq \frac{\bar{g} \varepsilon^{5 / 2}}{L^{\bar{\beta} / 2}\left(1-K_{*}\right)}+\frac{\bar{g}^{2}}{L^{\bar{\beta} / 2}}\left(\frac{1}{1-\frac{1}{L^{\beta / 2}}}\right)
$$




$$
\leq \frac{4 \bar{g}^{2}}{L^{\bar{\beta} / 2}}
$$

Moreover (ii a) $\Rightarrow$ (ii c), (integrate (ii a) with boundary condition $I_{n}(0)=0$ ).

Finally (ii) $\rightarrow$ (ii b) by the same reasoning.

The main job of this section is to prove the following:

Theorem 2. Suppose $v_{1}, v_{2}, \ldots, v_{n}$ satisfies Property $H_{n}$ then under RG iteration $v_{n+1}$ satisfies $H_{n+1}$.

Note that theorem 2 immediately implies Theorem 1:

Proof of Theorem 1 (given Theorem 2).

Since $v_{1}$ satisfies $H_{1}$ (Section IV, Proposition 1 ), Theorem $2 \Rightarrow v_{n}$ has the property $H_{n}$ for all $n$. In particular $H_{n}^{1}$ holds, all $n$. Since, $0<k_{*}<1$, (i) of $H_{n} \Rightarrow\left\{g_{n}\right\}$ is a sequence whose increments are absolutely summable, and hence Cauchy and (ia) says that every $g_{n}$ lies within an $\varepsilon^{3 / 2}$ ball of center $\bar{g}$. Hence $g_{n} \rightarrow g_{\infty}$ and

$$
\left|g_{\infty}-\bar{g}\right| \leq \varepsilon^{3 / 2}
$$

The decrease of $v_{*}$ in $\varphi^{2}$ beats the growth allowed in (ii b) and (ii c). From (iic), the $I_{n}$ are uniformly bounded in the $\|\cdot\|_{v_{*}}$ norm. $c^{(n-1)}$, see (ii), goes to zero as $n \rightarrow \infty$, and is summable. Now from (ii b) it follows that the $I_{n} \rightarrow I_{\infty}$ in the $\|\cdot\|_{v_{*}}$ norm, and

$$
\left\|I_{\infty}\right\|_{v_{*}} \leq \frac{4 \bar{g}^{2}}{L^{\bar{\beta} / 2}}
$$

So Theorem 1 has been proved (given Theorem 2)

The starting point for the proof of Theorem 2 will be the following formulae which give $\Delta v_{n}$ through the increments $\Delta g_{n}, \Delta I_{n}$.

To this end define:

$$
\begin{gathered}
A_{n}(\varphi) \doteq \sum_{l \geq 1} \frac{(-1)^{l}<v_{*}^{l}\left(g_{n}+I_{n}\right)^{l}>_{\varphi}}{l !} \\
L^{-\alpha} v_{*}(\varphi) \\
\Delta B_{n}(\varphi) \doteq \sum_{l \geq 1} \frac{(-1)^{l-1}}{l !} \sum_{l \leq m \leq l}\left(\begin{array}{c}
l \\
m
\end{array}\right) \frac{<v_{*}^{l}\left(g_{n-1}+I_{n-1}\right)^{l-m}\left(\Delta g_{n-1}+\Delta I_{n-1}\right)^{m}}{L^{-\alpha} v_{*}(\varphi)}
\end{gathered}
$$

Then we have: Increment formulae

$$
\Delta g_{n}=L^{\varepsilon}\left(1+L^{-\alpha} v_{*}(0) A_{n}(0)\right)^{-1} \Delta B_{n}(0)
$$




$$
\begin{gathered}
\frac{d}{d \varphi^{2}} \Delta I_{n}=L^{\varepsilon} \frac{d}{d \varphi^{2}}\left[\left(1+L^{-\alpha} v_{*} A_{n}\right)^{-1} \Delta B_{n}\right] \\
\Delta I_{n}(0)=0
\end{gathered}
$$

Note that the above make sense provided the series $(5.13-5.14)$ converge and $A_{n}$ is sufficiently small. This will be seen to be true presently because of the inductive hypothesis and $H_{n}$.

Proof of (5.15). Start from

$$
v_{n}=v_{*} \quad\left(g_{n}+I_{n}\right)
$$

and explicitly perfom the RG iteration $v_{n} \rightarrow v_{n+1}$. We get (replace $v_{0}=g_{0} v_{*}$ in (4.12) of section IV by $v_{n}$ )

$$
v_{n+1}(\varphi)=L^{D} \sum_{k \geq 1} \sum_{l_{1} \ldots l_{k}}(-1)^{|\underline{l}|-1} c_{k}(\underline{l}) \prod_{j=1}^{k}<v_{*}^{l_{j}}\left(g_{n}+I_{n}\right)^{l_{j}}>_{\varphi}
$$

and

$$
<F>_{\varphi}=\left(\mu_{\gamma} * F\right)\left(L^{\beta / 2} \varphi\right)
$$

Now write:

$$
g_{n}+I_{n}=\left(g_{n-1}+I_{n-1}\right)+\left(\Delta g_{n-1}+\Delta I_{n-1}\right)
$$

and expand binomially:

$$
\left(g_{n}+I_{n}\right)^{l_{j}}=\sum_{m_{j}=0}^{l_{j}}\left(\begin{array}{c}
l_{j} \\
m_{j}
\end{array}\right)\left(g_{n-1}+I_{n-1}\right)^{l_{j}-m_{j}}\left(\Delta g_{n-1}+\Delta I_{n-1}\right)^{m_{j}}
$$

If we insert this in $(* 1)$ above for each $\mathrm{j}$-factor, then the contribution corresponding to $m_{j}=0, j=1, \ldots, k$ gives us back $v_{n}$. Hence,

$$
\begin{gathered}
\Delta v_{n}=L^{D} \sum_{k \geq 1} \sum_{\substack{l_{1} \ldots l_{k} \\
l_{j} \geq 1}}(-1)^{|\underline{l}|-1} c_{k}(\underline{l}) \cdot \\
\cdot \sum_{\substack{m_{1} \ldots m_{k} \\
0 \leq n_{j} \leq l_{j}}}^{\prime} \prod_{j=1}^{k}\left(\begin{array}{c}
l_{j} \\
m_{j}
\end{array}\right)<v_{*}^{l_{j}}\left(g_{n-1}+I_{n-1}\right)^{l_{j}-m_{j}}\left(\Delta g_{n-1}+\Delta I_{n-1}\right)^{m_{j}}>
\end{gathered}
$$

where $\sum^{\prime}$ means at least one $m_{j} \geq 1$. We can write $\sum^{\prime}$ as

$$
\sum^{\prime}=k \sum_{\substack{m_{1} \ldots m_{k-1} \\ 0 \leq m_{j} \leq l_{j}}} \sum_{\substack{1 \leq m_{k} \leq l_{k}\\}}
$$


by symmetry. Note that

$$
k c_{k}(\underline{l})=\frac{(-1)^{k-1}}{\prod_{j=1}^{k} l_{j} !}
$$

from (4.13) of section 4 . With these replacements in $(* 2)$, divide each $\mathrm{j}$-factor there by $L^{-\alpha} v_{*}$, and compensate by multiplying within the k-sum by

$$
\left(L^{-\alpha} v_{*}\right)^{k}=L^{-\alpha} v_{*}\left(L^{-\alpha} v_{*}\right)^{k-1}
$$

The $L^{-\alpha} v_{*}$ can be factored out of (2) altogether, and $L^{D-\alpha} v_{*}=L^{\varepsilon} v_{*}$.

Performing the $l_{k}$ sum gives $\Delta B_{n}$. Each $l_{j}$ sum, for $j=1, \ldots, k-1$, gives the identical contribution $A_{n}$ (the binomial series can be summed up again). We thus get

$$
\Delta v_{n}=L^{\varepsilon} v_{*}\left(\sum_{k \geq 1} L^{-\alpha(k-1)} v_{*}^{k-1}(-1)^{k-1} A_{n}^{k-1}\right) \Delta B_{n}
$$

or

$$
\begin{gathered}
\Delta v_{n}=L^{\varepsilon} v_{*}\left(1+L^{-\alpha} v_{*} A_{n}\right)^{-1} \Delta B_{n} \\
=v_{*}\left[L^{\varepsilon}\left(1+L^{-\alpha} v_{*}(0) A_{n}(0)\right)^{-1} \Delta B_{n}(0)+\right. \\
\left.+\left\{L^{\varepsilon}\left(1+L^{-\alpha} v_{*} A_{n}\right)^{-1} \Delta B_{n}-(\text { same at } \varphi=0)\right\}\right] \\
=v_{*}\left[\Delta g_{n}+\Delta I_{n}\right]
\end{gathered}
$$

Formula (5.15) has been proved.

We have to give bounds on various quantities appearing on the RHS of the formulae given by (5.15) expressing the increments $\Delta g_{n}, \Delta I_{n}$. To obtain these bounds we shall make repeated use of the following Proposition 2, whose proof is given in the appendix A. This proposition gives a priori bounds on $R G$ integrals of the type we encounter.

Proposition 2.

Let $1 \leq q \leq l$ and $F(\varphi)$ a $\mu_{\gamma}$ integrable $C^{1}$ function of $\varphi^{2}$, satisfying

$$
F(0)=0
$$

and

$$
\left|\frac{d F}{d \varphi^{2}}\right| \leq c_{1} e^{\frac{\lambda_{*}}{2} L^{-\beta}|\varphi|^{2}}
$$

where $c_{1}>0$ is a constant. 
Then

$$
<v_{*}^{l} F^{q}>_{\varphi}=\left(\mu_{\gamma} *\left(v_{*}^{l} F\right)\right)\left(L^{\beta / 2} \varphi\right)
$$

is $C^{1}$ in $\varphi^{2}$, and there exists a constant $c_{2}>0$, independent of $L$, such that:

$$
\begin{gathered}
\text { (i) }\left|\frac{d}{d \varphi^{2}}\left(\frac{<v_{*}^{l} F^{q}>}{L^{-\alpha} v_{*}}\right)\right| \leq c_{1}^{q} c_{2} L^{-\beta} e^{\frac{\lambda_{*}}{2} L^{-\beta}|\varphi|^{2}} \\
\text { (ii) }\left|\frac{<v_{*}^{l} F^{q}>}{L^{-\alpha} v_{*}}\right|_{\varphi=0} \mid \leq c_{1}^{q} c_{2} \\
\text { (iii) }\left|\frac{<v_{*}^{l} F^{q}>}{L^{-\alpha} v_{*}}\right| \leq c_{1}^{q} c_{2}\left[L^{-\beta}|\varphi|^{2} e^{\frac{\lambda_{*}}{2}} L^{-\beta}|\varphi|^{2}+1\right]
\end{gathered}
$$

Remark. There is a trivial generalization of this proposition where we consider two functions $\mathrm{F}$ and $\mathrm{G}$ with above properties with $d_{1}$ appearing in (5.16), instead of $c_{1}$, for G. Instead of $F^{q}$, take $F^{q_{1}} G^{q_{2}}, 1 \leq q_{1}+q_{2} \leq l$. Then (i) - (iii) continue to hold with the replacement $c_{1}^{q} \rightarrow c_{1}^{q_{1}} d_{1}^{q_{2}}$, and with the same constant $c_{2}$ independent of L. Proof of Proposition 2.

This follows from Lemmas 1-6 in the appendix A.

\section{Define now:}

$$
F_{n, l, m, s}(\varphi) \doteq \frac{<v_{*}^{l}\left(g_{n-1}+I_{n-1}\right)^{l-m}(\Delta I)_{n-1}^{s}>_{\varphi}}{L^{-\alpha} v_{*}(\varphi)}
$$

for $1 \leq m \leq l, \quad 0 \leq s \leq m$.

These objects will obviously be encountered in bounding increments (5.15), (see (5.14)). Bounds on them are provided by the following Proposition 3, which follows immediately from Proposition 2 and Property $H_{n}$ of the inductive hypothesis.

Proposition 3:

Assume the inductive hypothesis with Property $H_{n}$.

Then there exists a constant $c_{3}=1+0\left(\varepsilon^{1 / 2}\right)$, such that

$$
\begin{gathered}
\text { (i) }\left|\frac{d F_{n, l, m, s}}{d \varphi^{2}}\right| \leq \frac{c_{2}}{L^{\beta}}\left(c^{(n-1)}\right)^{s}\left(c_{3}|\bar{g}|\right)^{(l-m)} e^{\frac{\lambda_{*}}{2} L^{-\beta}|\varphi|^{2}} \\
\text { (ii) }\left|F_{n, l, m, s}\right| \leq c_{2}\left(c^{(n-1)}\right)^{s}\left(c_{3}|\bar{g}|\right)^{l-m}\left[\frac{\varphi^{2}}{L^{\beta}} e^{\frac{\lambda_{*}}{2} L^{-\beta}|\varphi|^{2}}+1\right]
\end{gathered}
$$

Here $c_{2}$, independent of $L$, is the constant of Proposition 2.

Proof.

$$
\left|\frac{d F_{n, l, m, s}}{d \varphi^{2}}\right| \leq \sum_{p=0}^{l-m}\left(\begin{array}{c}
l-m \\
p
\end{array}\right)\left|g_{n-1}\right|^{p} \quad\left|\frac{d}{d \varphi^{2}} \frac{<v_{*}^{l} I_{n-1}^{(l-m)-p}\left(\Delta I_{n-1}\right)^{s}}{L^{-\alpha} v_{*}}\right|
$$


From part (i) of Proposition 2, (5.17) together with the remark following it, and part (ii) of Property $H_{n}$ (5.11), (ii a) of $H_{n}^{\prime}$ (5.12), the derivative term is bounded by:

$$
\frac{c_{2}}{L^{\beta}}\left(c^{(n-1)}\right)^{s}\left(\frac{4 \bar{g}^{2}}{L^{\bar{\beta} / 2}}\right)^{(l-m)-p} e^{\frac{\lambda_{*}}{2} L^{-\beta}|\varphi|^{2}}
$$

So the $R \cdot H \cdot S$ of $(*)$ is bounded by

$$
\frac{c_{2}}{L^{\beta}}\left(c^{(n-1)}\right)^{s}\left(\left|g_{n-1}\right|+\frac{4 \bar{g}^{2}}{L^{\bar{\beta} / 2}}\right)^{(l-m)} e^{\frac{\lambda_{*}}{2} L^{-\beta}|\varphi|^{2}}
$$

From part (ia) of $H_{n}^{\prime}(5.12)$,

$$
\left|g_{n-1}\right| \leq|\bar{g}|+\varepsilon^{3 / 2}
$$

Hence,

$$
\left|g_{n-1}\right|+\frac{4 \bar{g}^{2}}{L^{\bar{\beta} / 2}} \leq c_{3}|\bar{g}|
$$

where $c_{3}=1+0\left(\varepsilon^{1 / 2}\right)$, since $\bar{g}$ is $0(\varepsilon)$.

Part (ii) of Proposition 3 now follows.

Part (ii) follows in the same way, using part (iii) of Proposition 2, (5.19).

Proposition 3 has been proved.

Using Propositions 2 and 3 and property $H_{n}$ of the inductive hypothesis, we obtain easily bounds on $A_{n}$ and $\Delta B_{n}$ and its derivatives (appearing in (5.15)) summarized in the following proposition 4, whose proof is relegated to Appendix B.

$\underline{\text { Proposition } 4}$

$$
\begin{gathered}
\left|\left(1+L^{-\alpha} v_{*}(0) A_{n}(0)\right)^{-1}\right| \leq 1+L^{-\alpha}\left(\frac{\lambda}{2 \pi}\right)^{d / 2} \bar{g}+3 \varepsilon^{3 / 2} \\
\left|\frac{d A_{n}}{d \varphi^{2}}\right| \leq \frac{2 c_{2} c_{3}|\bar{g}|}{L^{\beta}} e^{\frac{\lambda_{*}}{2} L^{-\beta}|\varphi|^{2}} \\
\left|A_{n}\right| \leq 2 c_{2} c_{3}|\bar{g}|\left(\frac{\varphi^{2}}{L^{\beta}} e^{\frac{\lambda_{*}}{2} L^{-\beta}|\varphi|^{2}}+1\right) \\
\left|\Delta B_{n}(0)\right| \leq k_{*}^{n-1} \delta(\varepsilon)\left[1-\bar{g}\left(\frac{\lambda_{*}}{4 \pi}\right)^{d / 2}\left(1+\frac{1}{2} L^{-\beta}\right)\right. \\
\left.+c^{\prime} \frac{\bar{g}}{L^{\bar{\beta} / 2}}+5 \varepsilon^{3 / 2}\right] \\
L^{\varepsilon}\left|\frac{d}{d \varphi^{2}} \Delta B_{n}\right| \leq \frac{1}{4}\left[\frac{\bar{g}}{L^{\bar{\beta} / 2}} k_{*}^{n-1} \delta(\varepsilon)+\frac{\bar{g}^{2}}{L^{\bar{\beta} / 2}}\left(\frac{1}{L^{\bar{\beta} / 2}}\right)^{n}\right] \cdot e^{\frac{\lambda_{*}}{2} L^{-\beta}|\varphi|^{2}} \\
\left|\Delta B_{n}\right| \leq c\left[\bar{g} k_{*}^{n-1} \delta(\varepsilon)\left(1+\frac{1}{k_{*} L^{\bar{\beta} / 2}}\right)+\bar{g}^{2}\left(\frac{1}{L^{\bar{\beta} / 2}}\right)^{n}\right] . \\
\cdot\left(\frac{\varphi^{2}}{L^{\beta}} e^{\frac{\lambda_{*}}{2} L^{-\beta}|\varphi|^{2}}+1\right)
\end{gathered}
$$


The bounds proven in Proposition 4 will now enable us to prove Theorem 2.

Proof of Theorem 2

Claim 1

$$
\left|\Delta g_{n}\right| \cdot \leq k_{*}^{n} \delta(\varepsilon)
$$

This is part (i) of property $H_{n+1}$ see (5.11).

Proof: From (5.15), and the bounds $(5.23,5.26)$, we have

$$
\begin{aligned}
& \left|\Delta g_{n}\right| \leq L^{\varepsilon}\left|\left(1+L^{-\alpha} v_{*}(0) A_{n}(0)\right)^{-1}\right|\left|\Delta B_{n}(0)\right| \\
& \leq k_{*}^{n-1} \delta(\varepsilon) L^{\varepsilon}\left[1-\bar{g}\left(\frac{\lambda_{*}}{4 \pi}\right)^{d / 2}\left(1+\frac{1}{2} L^{-\beta}\right)+\frac{c^{\prime} \bar{g}}{L^{\bar{\beta} / 2}}+5 \varepsilon^{3 / 2}\right] \\
& \left.\dot{(1}+L^{-\alpha}\left(\frac{\lambda_{*}}{2 \pi}\right)^{d / 2} \bar{g}+3 \varepsilon^{3 / 2}\right) \\
& \leq k_{*}^{n-1} \delta(\varepsilon)\left\{L^{\varepsilon}\left[1-\bar{g}\left(\frac{\lambda_{*}}{4 \pi}\right)^{d / 2}\left(1+\frac{1}{2} L^{-\beta}-2^{d / 2} L^{-\alpha}\right)+9 \varepsilon^{3 / 2}\right]+\frac{L^{\varepsilon} c^{\prime} \bar{g}}{L^{\bar{\beta} / 2}}\right\}
\end{aligned}
$$

(we have used the fact, see 5.6a), that

$$
\bar{g}^{2}<\left(C \varepsilon^{1 / 4}\right) \varepsilon^{3 / 2}
$$

Thus

$$
\left|\Delta g_{n}\right| \leq k_{*}^{n-1} \delta(\varepsilon)\left\{L^{\varepsilon}\left[1-\left(-2 a_{1}\right) \bar{g}+9 \varepsilon^{3 / 2}\right]+\frac{L^{\varepsilon} c^{\prime} \bar{g}}{L^{\bar{\beta} / 2}}\right\}
$$

where $a_{1}$ is given by (4.25) of section IV. From the definition of the approximate fixed point $\bar{g}$, see (4.27) of Section IV:

$$
\bar{g}=\frac{L^{\varepsilon}-1}{L^{\varepsilon}\left(-a_{1}\right)}
$$

we get

$$
L^{\varepsilon}\left(1-\left(-2 a_{1}\right) \bar{g}\right)=2-L^{\varepsilon} \leq 1-\varepsilon \ln L
$$

Also, (see, (5.6))

$$
\begin{gathered}
L^{\varepsilon} \leq 1+C \varepsilon^{7 / 8} \\
L^{-\beta / 2} \leq \varepsilon^{5 / 2}
\end{gathered}
$$

and

$$
\bar{g}<C \varepsilon^{7 / 8}
$$

so that

$$
L^{\varepsilon}\left(9 \varepsilon^{3 / 2}+\frac{c^{\prime} \bar{g}}{L^{\bar{\beta} / 2}}\right)<10 \varepsilon^{3 / 2}
$$


Using (i), (ii) in $(*)$ above,

$$
\begin{gathered}
\left|\Delta g_{n}\right| \leq k_{*}^{n-1} \delta(\varepsilon)\left\{1-\varepsilon \ln L+10 \varepsilon^{3 / 2}\right\} \\
\leq k_{*}^{n} \delta(\varepsilon)
\end{gathered}
$$

by virtue of the definition (5.7). Claim 1 has been proved.

\section{Claim 2}

$$
\begin{gathered}
\left|\frac{d}{d \varphi} \Delta I_{n}\right| \leq c^{(n)} e^{\frac{\lambda_{*}}{2} L^{-\beta}|\varphi|^{2}} \\
c^{(n)}=\frac{\bar{g}}{L^{\bar{\beta} / 2}} k_{*}^{n-1} \delta(\varepsilon)+\bar{g}^{2}\left(\frac{1}{L^{\bar{\beta} / 2}}\right)^{n}
\end{gathered}
$$

This is part (ii) of Property $H_{n+1}$ (see 5.11)

Proof: Carrying out the derivative of the right hand side of the equation giving $\frac{d}{d \varphi^{2}} \Delta I_{n}$ in (5.15) gives:

$$
\left.\begin{array}{rl}
\left|\frac{d}{d \varphi^{2}} \Delta I_{n}\right| \leq & \left(1-L^{-\alpha} v_{*}\left|A_{n}\right|\right)^{-1}\left|L^{\varepsilon} \frac{d}{d \varphi^{2}} \Delta B_{n}\right|+ \\
& +\left\{\left(1-L^{-\alpha} v_{*}\left|A_{n}\right|\right)^{-2} L^{\varepsilon} L^{-\alpha}\left(\left|\frac{d v_{*}}{d \varphi^{2}} A_{n}\right|+v_{*}\left|\frac{d A_{n}}{d \varphi^{2}}\right|\right)\left|\Delta B_{n}\right|\right.
\end{array}\right\}
$$

and $\alpha \geq \beta$, for $d \geq 2$

From

$$
\begin{gathered}
v_{*}=\left(\frac{\lambda_{*}}{2 \pi}\right)^{d / 2} e^{-\frac{\lambda_{*}}{2}|\varphi|^{2}} \\
\frac{d v_{*}}{d \varphi^{2}}=-\frac{\lambda_{*}}{2} v_{*}
\end{gathered}
$$

and the bounds $(5.24,5.25)$ on $A_{n}$ and its derivative, we easily derive

$$
\begin{gathered}
L^{-\alpha} v_{*}\left|\frac{d A_{n}}{d \varphi^{2}}\right| \leq \frac{2 c_{2} c_{3}|\bar{g}|}{L^{2 \beta}} e^{-\frac{\lambda_{*}}{4}|\varphi|^{2}} \\
L^{-\alpha}\left|\frac{d v_{*}}{d \varphi^{2}} A_{n}\right| \leq \frac{8 c_{2} c_{3}|\bar{g}|}{L^{\beta}} e^{-\frac{\lambda_{*}}{4}|\varphi|^{2}} \\
L^{-\alpha} v_{*}\left|A_{n}\right| \leq \frac{4 c_{2} c_{3}|\bar{g}|}{L^{\beta}}
\end{gathered}
$$

Now we bound the various terms in $(* *)$ above.

1 Using (v) above and the bound (5.38) we get:

$$
\begin{gathered}
\left|\left(1-L^{-\alpha} v_{*}\left|A_{n}\right|\right)^{-1}\right| L^{\varepsilon} \frac{d}{d \varphi^{2}} \Delta B_{n}|| \\
\leq\left(1-\frac{4 c_{2} c_{3}|\bar{g}|}{L^{\beta}}\right)^{-1} \frac{1}{4}\left[\frac{\bar{g}}{L^{\bar{\beta} / 2}} k_{*}^{n-1} \delta(\varepsilon)+\frac{\bar{g}^{2}}{L^{\bar{\beta} / 2}}\left(\frac{1}{L^{\bar{\beta}} / 2}\right)^{n}\right] e^{\frac{\lambda_{*}}{2} L^{-\beta}}
\end{gathered}
$$




$$
\leq \frac{1}{2}\left[\frac{\bar{g}}{L^{\bar{\beta} / 2}} k_{*}^{n-1} \delta(\varepsilon)+\frac{\bar{g}^{2}}{L^{\bar{\beta} / 2}}\left(\frac{1}{L^{\bar{\beta} / 2}}\right)^{n}\right] e^{\frac{\lambda_{*}}{2} L^{-\beta}|\varphi|^{2}}
$$

$\underline{2}$ Next using (iii) and (iv) above, togethere with the bound (5.39) on $\Delta B_{n}$ gives for the term in braces \{\} in () above:

$$
\begin{aligned}
& |\{\}| \leq\left(1-\frac{4 c_{2} c_{3}|\bar{g}|}{L^{\beta}}\right)^{-1}\left(\frac{1}{L^{\bar{\beta}}}\right)\left(10 c_{2} c_{3} \bar{g}\right) e^{-\frac{\lambda_{*}}{4}|\varphi|^{2}} . \\
& \cdot 2 c\left(k_{*}^{n-1} \delta(\varepsilon) \bar{g}+\bar{g}^{2}\left(\frac{1}{L^{\bar{\beta} / 2}}\right)^{n}\right)\left(\frac{\varphi^{2}}{L^{\beta}} e^{\frac{\lambda_{*}}{2} L^{-\beta}|\varphi|^{2}}+1\right)
\end{aligned}
$$

We can trivially bound:

$$
e^{-\frac{\lambda_{*}}{4}\left|\varphi^{2}\right|}\left(\frac{\varphi^{2}}{L^{\beta}} e^{\frac{\lambda_{*}}{2} L^{-\beta}|\varphi|^{2}}+1\right) \leq c^{\prime} e^{\frac{\lambda_{*}}{2} L^{-\beta}|\varphi|^{2}}
$$

We plug this into $(* * *)$. Then from the overall factor $\left(\frac{1}{L^{\beta}}\right)$ in $(* * *)$ we use up $\frac{1}{L^{\beta / 2}}$ to bound all unnecessary constants by $1 / 2$. We then get

$$
|\{\}| \leq \frac{1}{2}\left[\frac{\bar{g}}{L^{\bar{\beta} / 2}} k_{*}^{n-1} \delta(\varepsilon)+\frac{\bar{g}^{2}}{L^{\bar{\beta} / 2}}\left(\frac{1}{L^{\bar{\beta} / 2}}\right)^{n}\right] e^{\frac{\lambda_{*}}{2} L^{-\beta}|\varphi|^{2}}
$$

Putting together this bound, together with that in 1$)$ above we get from $(* *)$

$$
\begin{gathered}
\left|\frac{d}{d \varphi^{2}}\right| \Delta I_{n} \| \leq\left[\frac{\bar{g}}{L^{\bar{\beta} / 2}} k_{*}^{n-1} \delta(\varepsilon)+\frac{\bar{g}^{2}}{L^{\bar{\beta} / 2}}\left(\frac{1}{L^{\bar{\beta} / 2}}\right)^{n}\right] e^{\frac{\lambda_{*}}{2} L^{-\beta}|\varphi|} \\
=c^{(n)} e^{\frac{\lambda_{*}}{2} L^{-\beta}|\varphi|^{2}}
\end{gathered}
$$

$\underline{\text { Claim } 2 \text { has been proved }}$

This completes the proof of Theorem 2.

As shown earlier Theorem $2 \Rightarrow$ Theorem 1 , and thus the scaling limit, in the hierarchical approximation, and convergence to a non-Gaussian fixed point has been proved.

\section{Acknowledgements}

We thank Dominique Mouhanna for bringing to our attention the work of David, Duplantier and Guitter. One of us (PKM) thanks David Brydges and Kristof Gawedzki for helpful conversations on the Renormalization Group. In particular, he thanks Krystof Gawedzki for discussions related to the subject of this paper. He thanks G.Jona-Lasinio and the Dipartimento di Fisica, Università di Roma - La Sapienza, where part of this work was done, for hospitality.

The other (M.C.) thanks the CNRS for support which made his visit to Paris possible and the Laboratoire de Physique Théorique et Hautes Energies, Université Paris VI, for hospitality. 


\section{$\underline{\text { Appendix A }}$}

This appendix is devoted to the proof of Proposition 2 of Section 5. First we state a useful representation. Let $F(\varphi)$ be a $\mu_{\gamma}$-integrable $C^{1}$ function of $\varphi^{2}$.

$$
<v_{*} F>_{\varphi} \doteq\left(\mu_{\gamma} *\left(v_{*} F\right)\right)\left(L^{\beta / 2} \varphi\right)
$$

Also, from Section IV and Gaussian integration

$$
<v_{*}>_{\varphi}=L^{-\alpha} v_{*}(\varphi)
$$

Thus,

$$
\frac{\left\langle v_{*} F>_{\varphi}\right.}{L^{-\alpha} v_{*}(\varphi)}=\frac{\int \frac{d^{d} \zeta}{(2 \pi \gamma)^{d / 2}} e^{-\frac{1}{2 \gamma}\left|\zeta-L^{\beta / 2} \varphi\right|^{2}} v_{*}(\zeta) F(\zeta)}{\int \frac{d^{d} \zeta}{(2 \pi \gamma)^{d / 2}} e^{-\frac{1}{2 \gamma}\left|\zeta-L^{\beta / 2} \varphi\right|^{2}} v_{*}(\zeta)}
$$

we can absorb,

$$
v_{*}(\zeta)=\left(\frac{\lambda_{*}}{2 \pi}\right)^{d / 2} e^{-\frac{\lambda_{*}}{2}|\zeta|^{2}}
$$

into the measure $d \mu_{\gamma}(\zeta)$ to obtain a new convolution kernel.

Infact,

$$
\begin{gathered}
\frac{d^{d} \zeta}{(2 \pi \gamma)^{d / 2}} e^{-\frac{1}{2 \gamma}\left|\zeta-L^{\beta / 2} \varphi\right|^{2}} v_{*}(\zeta) \\
=\left(\frac{\lambda_{*}}{2 \pi}\right)^{d / 2} \cdot \frac{1}{\left(1+\gamma \lambda_{*}\right)^{d / 2}} \frac{d^{d} \zeta}{(2 \pi \sigma)^{d / 2}} e^{-\frac{1}{2 \sigma}\left(\zeta-L^{-\beta / 2} \varphi\right)^{2}} \\
\cdot e^{-\frac{\left(1-L^{-\beta}\right)|\varphi|^{2}}{2 \sigma}}
\end{gathered}
$$

where

$$
\sigma=\left(\frac{1}{\gamma}+\lambda_{*}\right)^{-1}
$$

and we have used (Section IV), $1+\gamma \lambda_{*}=L^{\beta}$.

We therefore obtain:

$$
\frac{<v_{*} F>_{(\varphi)}}{L^{-\alpha} v_{*}(\varphi)}=\int d \mu_{\sigma}\left(\zeta-L^{-\beta / 2} \varphi\right) F(\zeta)
$$

where $\mu_{\sigma}$ is the Gaussian measure of covariance

$$
\sigma=\left(\frac{1}{\gamma}+\lambda_{*}\right)^{-1}=\frac{\gamma}{L^{\beta}}=0(1)
$$

The representation (A1) will be used in the following.

\section{$\underline{\text { Lemma } 1}$}


Let $F(\varphi)$ be a $\mu_{\sigma}$ integrable $C^{1}$ function of $\varphi^{2}$. Then,

$$
\begin{gathered}
\left|\frac{d}{d \varphi^{2}}\left(\frac{<v_{*} F>_{\varphi}}{L^{-\alpha} v_{*}(\varphi)}\right)\right| \\
\leq \frac{1}{2 \sigma} e^{-\frac{L^{-\beta}|\varphi|^{2}}{2 \sigma}} L^{-\beta} \sum_{j=0}^{\infty} \frac{1}{(2 j) !} \frac{\left(L^{-\beta}|\varphi|^{2}\right)^{j}}{\sigma^{2 j}} . \\
\cdot \int d \mu_{\sigma}(\zeta)\left[\left(\zeta_{1}^{2}\right)^{j}+\frac{\left(\zeta_{1}^{2}\right)^{j+1}}{(2 j+1) \sigma}\right] F(\zeta)
\end{gathered}
$$

Proof: By invariance of $\mathrm{F}$ and the measure, the $L \cdot H \cdot S \cdot$ of $(\mathrm{A} 1)$ is a function of $\varphi^{2}$. Apply

$$
\frac{d}{d \varphi^{2}}=\frac{1}{2|\varphi|^{2}} \vec{\varphi} \cdot \frac{\partial}{\partial \vec{\varphi}}
$$

to the integral kernel in (A1). We then obtain:

$$
\begin{aligned}
\frac{d}{d \varphi^{2}}\left(\frac{<v_{*} F>}{L^{-\alpha} v_{*}}\right) & =\frac{1}{2 \sigma}\left[\frac{1}{|\varphi|^{2}} \int \frac{d^{d} \zeta}{(2 \pi \sigma)^{d / 2}} e^{-\frac{\left(\zeta-L^{-\beta / 2} \varphi\right)^{2}}{2 \sigma}} L^{-\beta / 2} \vec{\varphi} \cdot \vec{\zeta} F(\zeta)\right. \\
& \left.-L^{-\beta} \int \frac{d^{d} \zeta}{(2 \pi \sigma)^{d / 2}} e^{-\frac{\left(\zeta-L^{-\beta / 2} \varphi\right)^{2}}{2 \sigma}} F(\zeta)\right]
\end{aligned}
$$

Since $\frac{\left\langle v_{*} F\right\rangle}{L^{-\alpha} v_{*}}$ is an inv. function of $\varphi^{2}$, we can choose coordinates:

$$
\begin{gathered}
\varphi=\left(\varphi_{1}, 0, \ldots, o\right),|\varphi|^{2}=\varphi^{2} \\
e^{-\frac{(\zeta-L-\beta / 2 \varphi)^{2}}{2 \sigma}}=e^{-\frac{L^{-\beta}|\varphi|^{2}}{2 \sigma}} e^{-\frac{|\zeta|^{2}}{2 \sigma}} e^{+L^{-\beta / 2 \frac{\varphi_{1} \zeta_{1}}{\sigma}}} \\
=e^{\frac{L^{-\beta}|\varphi|^{2}}{2 \sigma}} \sum_{j=0}^{\infty} \frac{1}{j !}\left(\frac{L^{-\beta / 2} \varphi_{1}}{\sigma}\right)^{j} e^{-\frac{|\zeta|^{2}}{2 \sigma}} \zeta_{1}^{j}
\end{gathered}
$$

Plugging $(*)$ in to $(3)$ we get:

$$
\frac{d}{d \varphi^{2}}\left(\frac{<v_{*} F>}{L^{-\alpha} v_{*}}\right)=\frac{1}{2 \sigma} e^{-\frac{L^{-\beta}|\varphi|^{2}}{2 \sigma}}\left(J_{1}-J_{2}\right)
$$

$\underline{\text { where }}$

$$
J_{1}=\frac{1}{|\varphi|^{2}} \sum_{j=0}^{\infty} \frac{1}{j !} \frac{1}{\sigma^{j}}\left(L^{-\beta / 2} \varphi_{1}\right)^{j+1} \int d \mu_{\sigma}(\zeta) \zeta_{1}^{j+1} F(\zeta)
$$

Since $F$ is inv. function, and $\mu_{\sigma}$ is even in $\zeta_{1}$, only odd $j \geq 1$ contribute. So with $j \rightarrow 2 j+1$,

$$
J_{1}=\frac{1}{|\varphi|^{2}} \sum_{j=0}^{\infty} \frac{1}{(j+1) !} \frac{1}{\sigma^{2 j+1}}\left(L^{-\beta / 2} \varphi_{1}\right)^{2 j+2} \int d \mu_{\sigma}(\zeta) \zeta_{1}^{2 j+1} F(\zeta)
$$




$$
=L^{-\beta} \sum_{j=0}^{\infty} \frac{1}{(2 j) !} \frac{\left(L^{-\beta}|\varphi|^{2}\right)^{j}}{\sigma^{2 j}} \int d \mu_{\sigma}(\zeta) \frac{\left(\zeta_{1}^{2}\right)^{j+1}}{\sigma(2 j+1)} F(\zeta)
$$

whereas,

$$
J_{2}=L^{-\beta} \sum_{j=0}^{\infty} \frac{1}{j !}\left(\frac{L^{-\beta / 2} \varphi_{1}}{\sigma}\right)^{j} \int d \mu_{\sigma}(\zeta) \zeta_{1}^{j} F(\zeta)
$$

Only even $j$ contribute, so $j \rightarrow 2 j$

$$
=L^{-\beta} \sum_{j=0}^{\infty} \frac{1}{(2 j) !} \frac{\left(L^{-\beta}|\varphi|^{2}\right)^{j}}{\sigma^{2 j}} \int d \mu_{\sigma}(\zeta)\left(\zeta_{1}^{2}\right)^{j} F(\zeta)
$$

Hence, from (A4), (A5), (A6) and we get:

$$
\begin{gathered}
\left|\frac{d}{d \varphi^{2}}\left(\frac{<v_{*}(F>}{L^{-\alpha} v_{*}}\right)\right| \leq \frac{1}{2 \sigma} e^{-\frac{L^{-\beta}|\varphi|^{2}}{2 \sigma}}\left(\text { vert } J_{1}|+| J_{2} \mid\right) \\
\leq \frac{1}{2 \sigma} e^{-\frac{L^{-\beta}|\varphi|^{2}}{2 \sigma}} L^{-\beta} \sum_{j=0}^{\infty} \frac{1}{(2 j) !} \frac{\left(L^{-\beta}|\varphi|^{2}\right)^{j}}{\sigma^{2 j}} . \\
\cdot \int d \mu_{\sigma}(\zeta)\left[\left(\zeta_{1}^{2}\right)^{j}+\frac{\left(\zeta_{1}^{2}\right)^{j+1}}{(2 j+1) \sigma}\right]|F(\zeta)|
\end{gathered}
$$

Thus Lemma 1 has been proved

Remark: Lemma 1 will now be applied in the following pages to special choices of the function F.

Lemma 2 Assume: $F(\varphi)$ is a $\mu_{\gamma}$ integrable $C^{1}$ function of $\varphi^{2}=|\varphi|^{2}, F(0)=0$ and

$$
\left|\frac{d F}{d \varphi^{2}}\right| \leq c_{1} e^{\frac{\lambda_{*}}{2} L^{-\beta}|\varphi|^{2}}
$$

Then,

$$
\left|\frac{d}{d \varphi^{2}}\left(\frac{<v_{*} F>}{L^{-\alpha} v_{*}}\right)\right| \leq c_{1} c_{2} L^{-\beta} e^{\frac{\lambda_{*}}{2} L^{-\beta}|\varphi|^{2}}
$$

for some constant $c_{2}>1, c_{2}$ is indept. of $L$

Proof: We shall use Lemma 1. Note that, from $\left(\mathrm{A} 8^{*}\right)$,

$$
|F| \leq c_{1}\left(\frac{2}{\lambda_{*}} L^{\beta}\right)\left(e^{\frac{\lambda_{*}}{2} L^{-\beta}|\varphi|^{2}}-1\right)
$$

Hence, from Lemma 1,

$$
\begin{aligned}
& \left|\frac{d}{d \varphi^{2}}\left(\frac{\left\langle v_{*} F>\right.}{L^{-\alpha} v_{*}}\right)\right| \leq \frac{c_{1}}{2 \sigma} L^{-\beta} \cdot\left(\frac{2}{\lambda_{*}}\right) e^{-\frac{L^{-\beta}|\varphi|^{2}}{2 \sigma}} L^{\beta} . \\
& \left\{\sum _ { j = 0 } ^ { \infty } \frac { 1 } { ( 2 j ) ! } \frac { ( L ^ { - \beta } | \varphi | ^ { 2 } ) ^ { j } } { \sigma ^ { 2 j } } \left[\left(f(1, j)+\frac{f(1, j+1)}{(2 j+1) \sigma}\right)\right.\right. \\
& \left.\left.\quad-\left(f(0, j)+\frac{f(0, j+1)}{(2 j+1) \sigma}\right)\right]\right\}
\end{aligned}
$$


where:

$$
\begin{gathered}
f(\alpha, j)=\int d \mu_{\sigma}(\zeta) e^{\frac{\lambda_{*}}{2} \alpha L^{-\beta}|\zeta|^{2}}\left(\zeta_{1}^{2}\right)^{j} \\
=\int \frac{d^{d} \zeta}{(2 \pi \sigma)^{d / 2}} e^{-\frac{\sigma}{2}\left(1-\frac{\lambda_{*} \alpha}{\sigma} L^{-\beta}\right)|\zeta|^{2}}\left(\zeta_{1}^{2}\right)^{j} \\
=\frac{1}{\left(1-\frac{\lambda_{*} \alpha}{\sigma} L^{-\beta}\right)^{d / 2}} \cdot \frac{\sigma^{j}}{\left(1-\frac{\lambda_{*} \alpha}{\sigma} L^{-\beta}\right)^{j}}(2 j-1) ! ! \\
(2 j-1) ! !=\frac{(2 j) !}{j ! 2^{j}}=(2 j-1)(2 j-3) \ldots 1
\end{gathered}
$$

Using, $\frac{(2(j+1)-1) ! !}{(2 j+1)}=(2 j-1) !$ we obtain:

$$
\begin{gathered}
f(\alpha, j)+\frac{f(\alpha, j+1)}{(2 j+1) \sigma}=\frac{1}{\left(1-\frac{\lambda_{*} \alpha}{\sigma} L^{-\beta}\right)^{d / 2}} \frac{\sigma^{j}}{\left(1-\frac{\lambda_{*} \alpha}{\sigma} L^{-\beta}\right)^{j}} \cdot \frac{(2 j) !}{j ! 2^{j}} . \\
\cdot\left[1+\frac{1}{\left(1-\frac{\lambda_{*} \alpha}{\sigma} L^{-\beta}\right)}\right] \\
{\left[f(\alpha, j)+\frac{f(\alpha, j+1)}{(2 j+1) \sigma}\right]-[\ldots]_{\alpha=0}} \\
=\frac{(2 j) !}{j ! 2^{j}} \cdot \sigma^{j}\left[\frac{1}{\left(1-\frac{\lambda_{*} \alpha}{\sigma} L^{-\beta}\right)^{d / 2}}\left(1+\frac{1}{\left(1-\frac{\lambda_{*} \alpha}{\sigma} L^{-\beta}\right)}\right)\left(\frac{1}{\left(1+\frac{\lambda_{*} \alpha}{\sigma} L^{-\beta}\right)^{j}}\right)-2\right]
\end{gathered}
$$

Hence the infinite sum in (A10)

$$
\begin{gathered}
\left\{\sum_{j=0}^{\infty} \ldots . .\right\}=e^{\frac{L^{-\beta}|\varphi|^{2}}{2 \sigma\left(1-\frac{\left.\lambda_{*} L^{-\beta}\right)}{\sigma}\right.}} \cdot \frac{1}{\left(1-\frac{\lambda_{*}}{\sigma} L^{-\beta}\right)^{d / 2}}\left(1+\frac{1}{\left(1-\frac{\lambda_{*}}{\sigma} L^{-\beta}\right)}\right) \\
-2 e^{\frac{L^{-\beta}|\varphi|^{2}}{2 \sigma}}
\end{gathered}
$$

We easily have

$$
\begin{gathered}
\frac{1}{\left(1-\frac{\lambda_{*}}{\sigma} L^{-\beta}\right)} \leq 1+2 \frac{\lambda_{*}}{\sigma} L^{-\beta} \\
1+\frac{1}{\left(1-\frac{\lambda_{*}}{\sigma} L^{-\beta}\right)} \leq 2\left(1+\frac{\lambda_{*}}{\sigma} L^{-\beta}\right)
\end{gathered}
$$

and

$$
1-\frac{1}{1-\frac{\lambda_{*}}{\sigma} L^{-\beta}}=-\frac{\lambda_{*}}{\sigma} L^{-\beta} \frac{1}{1-\frac{\lambda_{*}}{\sigma} L^{-\beta}}
$$

satisfies:

$$
-2 \frac{\lambda_{*}}{\sigma} L^{-\beta} \leq 1-\frac{1}{1-\frac{\lambda_{*}}{\sigma} L^{-\beta}} \leq-\frac{\lambda_{*}}{\sigma} L^{-\beta}
$$

Hence from (A13)

$$
\left\{\sum_{j=0}^{\infty} \ldots .\right\} \leq 2 e^{\frac{L-\beta|\varphi|^{2}}{2 \sigma\left(1-\frac{\lambda *}{\sigma} L^{-\beta}\right)}}\left[\left(1+\frac{4 \lambda_{*}}{\sigma} L^{-\beta}\right)-e^{\frac{-\lambda_{*}}{\sigma^{2}} L^{-2 \beta}|\varphi|^{2}}\right]
$$


and, since

$$
\begin{gathered}
1-e^{-\frac{\lambda_{*}}{\sigma^{2}} L^{-2 \beta}|\varphi|^{2}} \leq \frac{\lambda_{*}}{\sigma^{2}} L^{-2 \beta}|\varphi|^{2} \\
\leq L^{-\beta}\left(\frac{1}{\delta}\right) e^{\delta \frac{\lambda_{*}}{\sigma^{2}} L^{-\beta}|\varphi|^{2}}
\end{gathered}
$$

where $\delta$ has to be chosen.

$$
\left\{\sum_{j=0}^{\infty} \ldots .\right\} \leq 2 L^{-\beta} e^{\frac{L^{-\beta}|\varphi|^{2}}{2 \sigma\left(1-\frac{\lambda_{*}}{\sigma} L^{-\beta}\right)}}\left[4 \frac{\lambda_{*}}{\sigma}+\frac{1}{\delta} e^{\delta \frac{\lambda_{*}}{\sigma^{2}} l^{-\beta}|\varphi|^{2}}\right]
$$

Plugging the bound (A14) in (A10) we get:

$$
\begin{gathered}
\left|\frac{d}{d \varphi^{2}}\left(\frac{<v_{*} F>}{L^{-\alpha} v_{*}}\right)\right| \\
\leq \frac{c_{1}}{2 \sigma} L^{-\beta} \frac{2}{\lambda_{*}} \cdot(2) e^{\frac{\left(\lambda_{*}\right) L^{-2 \beta}}{\sigma^{2}}|\varphi|^{2}}\left[4 \frac{\lambda_{*}}{\sigma}+\frac{1}{\delta} e^{\delta \frac{\lambda_{*}}{\sigma^{2}} L^{-\beta}|\varphi|^{2}}\right] \\
\leq \frac{2 c_{1}}{\left(\lambda_{*}\right) \sigma} L^{-\beta}\left[4\left(\frac{\lambda_{*}}{\sigma}\right) e^{\frac{\lambda_{*}}{2} L^{-\beta}|\varphi|^{2}}+\frac{1}{\delta} e^{\frac{\lambda_{*}}{2} L^{-\beta}|\varphi|^{2}}\left(2 \frac{\delta^{2}}{\sigma^{2}}+\frac{2 L^{-\beta}}{\sigma^{2}}\right)\right]
\end{gathered}
$$

Choose: $\delta=\frac{\sigma^{2}}{2}\left(1-\frac{1}{2}\right)=\frac{\sigma^{2}}{4}$. so that for L, suff. large since $\sigma=0(1)$

$$
\left(\frac{2 \delta}{\sigma^{2}}+\frac{2 L^{-\beta}}{\sigma^{2}}\right)=1-1 / 2+\frac{2 L^{-\beta}}{\sigma^{2}}<1
$$

and hence

$$
\left|\frac{d}{d \varphi^{2}}\left(\frac{<v_{*} F>}{L^{-\alpha} v_{*}}\right)\right| \leq \frac{2 c_{1}}{\left(\lambda_{*}\right) \sigma} 4\left(\frac{\lambda_{*}}{\sigma}+\frac{1}{\sigma^{2}}\right) e^{\frac{\lambda_{*}}{2} L^{-\beta}|\varphi|^{2}} \cdot L^{-\beta}
$$

$\underline{\text { Choose }} c_{2}=\frac{8}{\sigma^{2}}\left(1+\frac{1}{\sigma \lambda_{*}}\right)>1$

Then:

$$
\left|\frac{d}{d \varphi^{2}}\left(\frac{<v_{*} F>}{L^{-\alpha} v_{*}}\right)\right| \leq c_{1} c_{2} L^{-\beta} e^{\frac{\lambda_{*}}{2} L^{-\beta}|\varphi|^{2}}
$$

and Lemma 2 has been proved.

\section{$\underline{\text { Lemma } 3}$}

For $1 \leq q \leq l, l \geq 2$

$$
\left|\frac{d}{d \varphi^{2}} \frac{<v_{*}^{l} F^{q}>}{L^{-\alpha} v_{*}}\right| \leq c_{1}^{q} L^{-\beta} e^{\frac{\lambda_{*}}{2} L^{-\beta}|\varphi|^{2}}
$$

where F satisfies the hypothesis of Lemma 2

\section{$\underline{\text { Proof }}$}

Writing,

$$
\frac{<v_{*}^{l} F^{q}>}{L^{-\alpha} v_{*}}=\frac{<v_{*}\left(v_{*}^{l-1} F^{q}\right)>}{L^{-\alpha} v_{*}}
$$


apply Lemma 1 , choosing for $\mathrm{F},\left(v_{*}^{l-1} F^{q}\right)$ Then

$$
\begin{gathered}
\left|\frac{d}{d \varphi^{2}} \frac{<v_{*}^{l} F^{q}>}{L^{-\alpha} v_{*}}\right| \leq \frac{1}{2 \sigma} e^{-\frac{L^{-\beta}|\varphi|^{2}}{2 \sigma}} L^{-\beta} . \\
\sum_{j=0}^{\infty} \frac{1}{(2 j) !} \frac{\left(L^{-\beta}|\varphi|^{2}\right)^{j}}{\sigma^{2} j} \int d \mu_{\sigma}(\zeta)\left[\left(\zeta_{1}^{2}\right)^{j}+\frac{\left(\zeta_{1}^{2}\right)^{j+1}}{\sigma(2 j+1)}\right]\left|\left(v_{*}^{l-1} F^{q}\right)(\zeta)\right| \\
v_{*}^{l-1}=\left(\frac{\lambda_{*}}{2 \pi}\right)^{(l-1) \frac{d}{2}} e^{-\frac{\lambda_{*}}{2}(l-1)|\varphi|^{2}}
\end{gathered}
$$

From $\left(\mathrm{A} 9^{*}\right)$, in the bound on $F(\zeta)$ following the hypothesis of Lemma 2,

$$
\begin{gathered}
|F(\varphi)| \leq c_{1} \cdot\left(\frac{2}{\lambda_{*}} L^{\beta}\right) e^{\frac{\lambda_{*}}{2} L^{-\beta}|\varphi|^{2}}\left(1-e^{\left.-\frac{\lambda_{*}}{2} L^{-\beta}|\varphi|^{2}\right)}\right. \\
\leq c_{1} \cdot e^{\frac{\lambda_{*}}{2} L^{-\beta} \cdot|\varphi|^{2}} \\
\leq c_{1} \cdot \frac{2}{\lambda_{*} \delta} \cdot e^{\frac{\lambda_{*}}{2}\left(\delta+L^{-\beta}\right)|\varphi|^{2}}
\end{gathered}
$$

where $\delta$ is to chosen, $\delta>0$

Hence

$$
\begin{gathered}
\left|v_{*}^{l-1}(\zeta) F^{q}(\zeta)\right| \leq c_{1}^{q}\left[\frac{2}{\lambda_{*} \delta}\left(\frac{\lambda_{*}}{2 \pi}\right)^{(l-1) \frac{d}{2}}\right]^{q} \\
e^{-\frac{\lambda_{*}}{2}\left[(l-1)-q\left(\delta+L^{-\beta}\right)\right]|\zeta|^{2}}
\end{gathered}
$$

Choose:

$$
\delta=\frac{1}{2}-L^{-\beta}
$$

Then,

$$
\begin{gathered}
(l-1)-q\left(\delta+L^{-\beta}\right) \geq(l-1)-l\left(\delta+L^{-\beta}\right) \text { since } q \leq l \\
=l\left[1-\frac{1}{l}-\delta-L^{-\beta}\right] \\
=l\left[\frac{1}{2}-\frac{1}{l}\right] \geq 0, \text { since } l \geq 2
\end{gathered}
$$

Hence, $(l-1)-q\left(\delta+L^{-\beta}\right) \geq 0$ Using this in (A17), get:

$$
\left|v_{*}^{l-1}(\zeta) F^{q}(\zeta)\right| \leq c_{1}^{q}\left[\frac{4}{\lambda_{*}\left(1-2 L^{-\beta}\right)}\left(\frac{\lambda_{*}}{2 \pi}\right)^{\frac{d}{2}}\right]^{q}
$$

(have used $0<\lambda_{*} \leq 1$, as is easy to show).

Using the bound (A19) we have from (A16)

$$
\left|\frac{d}{d \varphi^{2}} \frac{<v_{*}^{l} F^{q}>}{L^{-\alpha} v_{*}}\right| \leq \frac{1}{2 \sigma} e^{-\frac{L^{-\beta}|\varphi|^{2}}{2 \sigma}} L^{-\beta} c_{1}^{q}\left[\frac{4\left(\frac{\lambda_{*}}{2 \pi}\right)^{\frac{d}{2}}}{\lambda_{*}\left(1-2 L^{-\beta}\right)}\right]^{q}
$$




$$
\cdot \sum_{j=0}^{\infty}\left\{\frac{1}{(2 j) !} \frac{\left(L^{-\beta}|\varphi|^{2}\right)^{j}}{\sigma^{2 j}} \int d \mu_{\sigma}(\zeta)\left[\left(\zeta_{1}^{2}\right)^{j} \frac{\left(\zeta_{1}^{2}\right)^{j+1}}{(2 j+1)^{\sigma}}\right]\right\}
$$

The integral is computed as in the proof of Lemma 2, and we get:

$$
2 \cdot \frac{(2 j) !}{j 2^{j}} \sigma^{j}
$$

so that the sum

$$
\sum_{j=0}^{\infty}\{\}=2 e^{\frac{L^{-\beta}|\varphi|^{2}}{2 \sigma}}
$$

We then get from (A20)

$$
\left|\frac{d}{d \varphi^{2}} \frac{<v_{*}^{l} F^{q}>}{L^{-\alpha} v_{*}}\right| \leq c_{1}^{q} L^{-\beta}\left\{\frac{1}{\sigma}\left[\frac{4\left(\frac{\lambda_{*}}{2 \pi}\right)^{d / 2}}{\lambda_{*}\left(1-2 L^{-\beta}\right)}\right]^{q}\right\}
$$

Look at the constant in \{\} . Since $0<\lambda_{*} \leq 1, d \geq 3$

$$
\{\} \leq \frac{1}{\sigma}\left[\frac{4}{(2 \pi)^{3 / 2}} \cdot \frac{1}{\left(1-2 L^{-\beta}\right)}\right]^{q} \leq 1
$$

$$
1 \leq q \leq l
$$

since, $\sigma=\frac{\gamma}{L^{\beta}}=2^{\beta / 2} \frac{\left(1-L^{-\beta}\right)}{\beta}$, (and this is regular as $\beta \rightarrow 0$ ). Hence:

$$
\begin{gathered}
\left|\frac{d}{d \varphi^{2}} \frac{<v_{*}^{l} F^{q}>}{L^{-\alpha} v_{*}}\right| \leq c_{1}^{q} L^{-\beta} \\
\leq c_{1}^{q} L^{-\beta} e^{\frac{\lambda_{*}}{2} L^{-\beta}|\varphi|^{2}}
\end{gathered}
$$

and Lemma 3 has been proved.

\section{Remark.}

We can collect Lemma 2 and Lemma 3 into a single Lemma.

\section{$\underline{\text { Lemma } 4}$}

Let $1 \leq q \leq l$, and $F(\varphi)$ a $\mu_{\gamma}$ integrable $C^{1}$ function in $\varphi^{2}$, satisfying the bound

$$
\left|\frac{d F}{d \varphi^{2}}\right| \leq c_{1} e^{\frac{\lambda_{*}}{2}|\varphi|^{2}}
$$

Then $\exists c_{2}>1$, independent of $\mathrm{L}$, such that

$$
\left|\frac{d}{d \varphi^{2}}\left(\frac{<v_{*}^{l} F^{q}>}{L^{-\alpha} v_{*}}\right)\right| \leq c_{1}^{q} c_{2} L^{-\beta} e^{\frac{\lambda_{*}}{2} L^{-\beta}|\varphi|^{2}}
$$

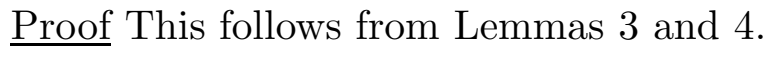


We need two further elementary bounds.

\section{$\underline{\text { Lemma } 5}$}

Let $\mathrm{F}$ satisfy the conditions of Lemma 2, which are the same in Lemmas 2-4, Then, for $1 \leq q \leq l$, there exists a constant $c_{2}$ independent of $L$ such that

$$
\left|\frac{<v_{*}^{l} F^{q}>_{\varphi}}{L^{-\alpha} v_{*}(\varphi)}\right|_{\varphi=0} \mid \leq c_{1}^{q} c_{2}
$$

$\underline{\text { Proof }}$

$$
\left|\frac{<v_{*}^{l} F^{q}>_{\varphi}}{L^{-\alpha} v_{*}(\varphi)}\right|_{\varphi=0}\left|\leq \int d \mu_{\sigma}(\zeta) v_{*}^{l-1}(\zeta)\right| F^{q}(\zeta) \mid
$$

(i) $l=q=1$. Then, using $\left(\mathrm{A} 9^{*}\right)$,

$$
\begin{gathered}
\int d \mu_{\sigma}(\zeta)|F(\zeta)| \leq c_{1}\left(\frac{2}{\lambda_{*}} L^{\beta}\right)\left[\left(\int d \mu_{\sigma}(\zeta) e^{\frac{\lambda_{*}}{2} L^{-\beta}|\zeta|^{2}}\right)-1\right] \\
=c_{1}\left(\frac{2}{\lambda_{*}} L^{\beta}\right)\left[\left(1-\frac{\lambda_{*}}{\sigma} L^{-\beta}\right)^{d / 2}-1\right] \\
\leq c_{1} c_{2}
\end{gathered}
$$

(ii) $1 \leq q \leq l, l \geq 2$. use (A19) and (*), between (A20) and (A21), to get

$$
\int d \mu_{\sigma}(\zeta)\left|v_{*}^{l-1} F^{q}(\zeta)\right| \leq c_{1}^{q} c_{2}
$$

Putting (i) and (ii) together we get (A23). Lemma 5 has been proved.

Finally integrate (A22) from 0 to $\varphi^{2}$ and use (A23) to obtain:

$\underline{\text { Lemma } 6}$

$$
\left|\frac{<v_{*}^{l} F^{q}>_{\varphi}}{L^{-\alpha} v_{*}(\varphi)}\right| \leq c_{1}^{q} c_{2}\left[L^{-\beta}|\varphi|^{2} e^{\frac{\lambda_{*}}{2} L^{-\beta}|\varphi|^{2}}+1\right]
$$

Lemma 4,5,6 take care of Proposition 2.

Proposition 2 has been proved. The remark after Proposition 2 is a trivial extension of the above.

\section{Appendix B}

Proof of Proposition 4

1. Bounds on $A_{n}$ and derivatives

Starting from (5.13) we can write:

$$
\begin{gathered}
A_{n}(0)=-\left.\frac{<V_{*}\left(g_{n}+I_{n}\right)>}{L^{-\alpha} v_{*}}\right|_{\varphi=0}+R_{1} \\
=-g_{n}-\left.\frac{<v_{*} I_{n}>}{L^{-\alpha} v_{*}}\right|_{\varphi=0}+R_{1}
\end{gathered}
$$


where,

$$
R_{1}=\left.\sum_{l \geq 2} \frac{(-1)^{l}}{l !} \frac{<v_{*}^{l}\left(g_{n}+I_{n}\right)^{l}>}{L^{-\alpha} v_{*}}\right|_{\varphi=0}
$$

Hence,

$$
\left|A_{n}(0)+g_{n}\right| \leq\left|\frac{<v_{*} I_{n}>}{L^{-\alpha} v_{*}}\right|_{\varphi=0}|+| R_{1} \mid
$$

The first term on $R \cdot H \cdot S$ is bounded above by:

$$
c_{2} \frac{4 \bar{g}^{2}}{L^{\bar{\beta} / 2}}
$$

(use part (ii), 5.18, of Proposition 2 and (ii a) of property $H_{n}^{1}(5.12)$ ).

On the other hand,

$$
\begin{aligned}
\left|R_{1}\right| \leq \sum_{l \geq 2} \frac{1}{l !} \sum_{m=0}^{l}\left(\begin{array}{c}
l \\
m
\end{array}\right)\left|g_{n}\right|^{l-m}\left|\frac{<v_{*}^{l} I_{n}^{m}>}{L^{-\alpha} v_{*}}\right|_{\varphi=0} \mid \\
\leq \sum_{l \geq 2} \frac{1}{l !} \sum_{m=0}^{l}\left(\begin{array}{c}
l \\
m
\end{array}\right)\left|g_{n}\right|^{l-m} c_{2}\left(\frac{4 \bar{g}^{2}}{L^{\bar{\beta} / 2}}\right)^{m} \\
=c_{2} \sum_{l \geq 2} \frac{1}{l !}\left(\left|g_{n}\right|+\frac{4 \bar{g}^{2}}{L^{\bar{\beta} / 2}}\right)^{l}
\end{aligned}
$$

Now the serie converges. Use (ia) of proporty $H_{n} 1$ (5.12) to deduce:

$$
\left|R_{1}\right| \leq 2 c_{2} \bar{g}^{2}
$$

Hence,

$$
\left|A_{n}(0)+g_{n}\right| \leq 6 c_{2} \bar{g}^{2}
$$

so, using (ia) of $H_{n}^{\prime}$, get

$$
-\bar{g}-2 \varepsilon^{3 / 2} \leq A_{n}(0) \leq-\bar{g}+2 \varepsilon^{3 / 2}
$$

Finally, from above and

$$
\left(1+L^{-\alpha} v_{*} A_{n}\right)^{-1}=1-L^{-\alpha} v_{*} A_{n}+\frac{\left(L^{-\alpha} v_{*} A_{n}\right)^{2}}{1+L^{-\alpha} v_{*} A_{n}}
$$

deduce,

$$
\left|\left(1+L^{-\alpha} v_{*}(0) A_{n}(0)\right)^{-1}\right| \leq 1+L^{-\alpha}\left(\frac{\lambda_{*}}{2 \pi}\right)^{d / 2} \bar{g}+3 \varepsilon^{3 / 2}
$$

(B1) is just (5.23). 
Next we bound: $\frac{d A n}{d \varphi^{2}}$. Starting from $(5.13)$

$$
\left|\frac{d A n}{d \varphi^{2}}\right| \leq \sum_{l \geq 1} \frac{1}{l !} \sum_{m=0}^{l}\left(\begin{array}{c}
l \\
m
\end{array}\right)\left|g_{n}\right|^{l-m}\left|\frac{d}{d \varphi^{2}} \frac{<v_{*}^{l} I_{n}^{m}>}{L^{-\alpha} v_{*}}\right|
$$

Use proposition 2 and (ii a), 5,12, of property $H_{n}^{\prime}$, by the inductive hypothesis. Then:

$$
\begin{gathered}
\left|\frac{d A n}{d \varphi^{2}}\right| \leq \sum_{l \geq 1} \frac{1}{l !} \sum_{m=0}^{l}\left(\begin{array}{c}
l \\
m
\end{array}\right)\left|g_{n}\right|^{l-m} \frac{c_{2}}{L^{\beta}}\left(\frac{4 \bar{g}^{2}}{L^{\bar{\beta}} / 2}\right)^{l} e^{\frac{\lambda_{*}}{2} L^{-\beta}|\varphi|^{2}}= \\
\frac{c_{2}}{L^{\beta}} \sum_{l \geq 1} \frac{1}{l !}\left(\left|g_{n}\right|+\frac{4 \bar{g}^{2}}{L^{\bar{\beta} / 2}}\right)^{l} e^{\frac{\lambda_{*}}{2} L^{-\beta}}
\end{gathered}
$$

The series converges. Now use (ia) of $H_{n}^{\prime}, 5.12$, to deduce

$$
\left|\frac{d A n}{d \varphi^{2}}\right| \leq \frac{2 c_{2} c_{3}|\bar{g}|}{L^{\beta}} e^{\frac{\lambda_{*}}{2} L^{-\beta} \varphi^{2}}
$$

Finally, using part (iii) of Proposition 2, (5.19), and $H_{n}^{\prime}$ we get in the same way:

$$
\left|A_{n}\right| \leq 2 c_{2} c_{3}|\bar{g}|\left(\frac{\varphi^{2}}{L^{\beta}} e^{\frac{\lambda_{*}}{2} L^{-\beta}}|\varphi|^{2}+1\right)
$$

(B2) and (B3) are (5.24) and (5.25) respectively.

2. Bounds on $\Delta B_{n}$ and derivatives

In (5.14) we make the binomial expansion:

$$
\left(\Delta g_{n-1}+\Delta I_{n-1}\right)^{m}=\sum_{s=0}^{m}\left(\begin{array}{c}
m \\
s
\end{array}\right)\left(\Delta g_{n-1}\right)^{m-s}\left(\Delta I_{n-1}\right)^{s}
$$

to obtain:

$$
\Delta B_{n}=\sum_{l \geq 1} \frac{(-1)^{l-1}}{l !} \sum_{1 \leq m \leq l}\left(\begin{array}{c}
l \\
m
\end{array}\right) \sum_{s=0}^{m}\left(\begin{array}{c}
m \\
s
\end{array}\right)\left(\Delta g_{n-1}\right)^{m-s} F_{n, l, m, s}
$$

where $F_{n, l, m, s}$ is given by $(5.20)$.

We separate out the contribution of the terms with $s=0$, (called $\left.\Delta C_{n}\right)$, and the contribution $s \geq 1$, (called $\left.\Delta D_{n}\right)$. $\Delta D_{n}$ has thus at least one irrelevant term $\Delta I_{n-1}$. We get

$$
\begin{aligned}
& \Delta C_{n}=\left(\Delta g_{n-1}\right) \sum_{l \geq 1} \frac{(-1)^{l-1}}{l !} \sum_{1 \leq m \leq l}\left(\begin{array}{c}
l \\
m
\end{array}\right)\left(\Delta g_{n-1}\right)^{m-1} F_{n, l, m, 0} \\
& =\left(\Delta g_{n-1}\right)\left[\sum_{l \geq 1} \frac{(-1)^{l-1}}{(l-1) !} F_{n, l, 1,0}+\right. \\
& \left.\left(\Delta g_{n-1}\right) \sum_{l \geq 2} \frac{(-1)^{l-1}}{l !} \sum_{2 \leq m \leq l}\left(\begin{array}{c}
l \\
m
\end{array}\right)\left(\Delta g_{n-1}\right)^{m-2} F_{n, l, m, 0}\right]
\end{aligned}
$$


and

$$
\Delta D_{n}=\sum_{l \leq 1} \frac{(-1)^{l-1}}{l !} \sum_{1 \leq m \leq l}\left(\begin{array}{c}
l \\
m
\end{array}\right) \sum_{s=1}^{m}\left(\begin{array}{c}
m \\
s
\end{array}\right)\left(\Delta g_{n-1}\right)^{m-s} F_{n, l, m, s} \quad B(6)
$$

and

$$
\Delta B_{n}=\Delta C_{n}+\Delta D_{n}
$$

First we give a bound on $\Delta C_{n}(0)$, which we write (see B 5 ),

$$
\Delta C_{n}(0)=\left(\Delta g_{n-1}\right)\left[1-F_{n, 2,1,0}(0)+R_{2}+R_{3}\right]
$$

where

$$
\begin{gathered}
R_{2}=\sum_{l \geq 3} \frac{(-1)^{l-1}}{(l-1) !} F_{n, l, 1,0}(0) \\
R_{3}=\Delta g_{n-1} \sum_{l \geq 2} \frac{(-1)^{l-1}}{l !} \sum_{2 \leq m \leq l}\left(\begin{array}{c}
l \\
m
\end{array}\right)\left(\Delta g_{n-1}\right)^{m-2} F_{n, l, m, 0}(0)
\end{gathered}
$$

Now,

$$
\begin{gathered}
F_{n, 2,1,0}(0)=\left.\frac{<v_{*}^{2}\left(g_{n-1}+I_{n-1}\right)>}{L^{-\alpha} v_{*}}\right|_{\varphi=0} \\
=g_{n-1}\left(\frac{\lambda_{*}}{4 \pi}\right)^{d / 2}\left(1+\frac{1}{2} L^{-\beta}\right)+\left.\frac{<v_{*}^{2} I_{n-1}>}{L^{-\alpha} v_{*}}\right|_{\varphi=0}
\end{gathered}
$$

From the inductive hypothesis and (i a) of $H_{n}^{\prime}$ (5.12),

$$
\left|g_{n-1}-\bar{g}\right| \leq \varepsilon^{3 / 2}
$$

From (ii a) of $H_{n}^{\prime}$ and part (i), 5.17, of Proposition 2,

$$
\left|\frac{<v_{*}^{2} I_{n-1}>}{L^{-\alpha} v_{*}}\right|_{\varphi=0} \mid \leq c_{2} \frac{4 \bar{g}^{2}}{L^{\bar{\beta} / 2}}
$$

Hence

$$
\left|1-F_{m, 2,1,0}(0)\right| \leq 1-\bar{g}\left(\frac{\lambda_{*}}{4 \pi}\right)^{d / 2}\left(1+\frac{1}{2} L^{-\beta}\right)+2 \varepsilon^{3 / 2}
$$

We have from part (ii), (5.22), of Proposition 3,

$$
\begin{gathered}
\left|R_{2}\right| \leq \sum_{l \geq 3} \frac{1}{(l-1) !}\left|F_{n, l, 1,0}(0)\right| \\
\leq c_{2} \sum_{l \geq 3} \frac{1}{(l-1) !}\left(c_{3}|\bar{g}|\right)^{l-1} \leq 2 c_{2}\left(c_{3} \bar{g}\right)^{2} \\
\leq 2 \varepsilon^{3 / 2}
\end{gathered}
$$

since the series converges. 
Next, always using Proposition 3, and part (i), (5.11), of property $H_{n}$ of inductive hypothesis:

$$
\begin{gathered}
\left|R_{3}\right| \leq\left|\Delta g_{n-1}\right| \sum_{l \geq 2} \frac{1}{l !} \sum_{2 \leq m \leq l}\left(\begin{array}{c}
l \\
m
\end{array}\right)\left|\Delta g_{n-1}\right|^{m-2}\left|F_{n, l, n, 0}(0)\right| \\
\leq k_{*}^{n-1} \delta(\epsilon) \sum_{l \geq 2} \frac{1}{l !} \sum_{m=2}^{l}\left(\begin{array}{c}
l \\
m
\end{array}\right)\left(k_{*}^{n-1} \delta(\varepsilon)\right)^{m-2}\left(c_{3}|\bar{g}|\right)^{l-m} \\
\leq 2 k_{*}^{n-1} \delta(\varepsilon) \\
\leq \varepsilon^{3 / 2}
\end{gathered}
$$

since the series converges. Using the above bounds we get:

$$
\left|\Delta C_{n}(0)\right| \leq\left|\Delta g_{n-1}\right|\left[\left|1-F_{n, 2,1,0}^{(0)}\right|+\left|R_{2}\right|+\left|R_{3}\right|\right]
$$

or

$$
\left|\Delta C_{n}(0)\right| \leq k_{*}^{n-1} \delta(\varepsilon)\left[1-\bar{g}\left(\frac{\lambda_{*}}{4 \pi}\right)^{d / 2}\left(1+\frac{1}{2} L^{-\beta}\right)+5 \varepsilon^{3 / 2}\right]
$$

Next we bound the derivative of $\Delta C_{n}$.

Starting from (B5),

$$
\begin{aligned}
& \left|\frac{d}{d \varphi^{2}} \Delta C_{n}\right| \leq\left|\Delta g_{n-1}\right|\left[\sum_{l \geq 2} \frac{1}{(l-1) !}\left|\frac{d}{d \varphi^{2}} F_{n, l, 1,0}\right|+\right. \\
& +\left|\Delta g_{n-1}\right| \sum_{l \geq 2} \frac{1}{l !} \sum_{m=2}^{l}\left(\begin{array}{c}
l \\
m
\end{array}\right)\left|\Delta g_{n-1}\right|^{m-2} \\
& \left.\cdot\left|\frac{d}{d \varphi^{2}} F_{n, l, m, o}\right|\right]
\end{aligned}
$$

Now use part (i), (5.21), of Proposition 3 and from inductive hypothesis part (i) of $H_{n},(5.11)$, to get:

$$
\begin{aligned}
& \left|\frac{d}{d \varphi^{2}} \Delta C_{n}\right| \leq k_{*}^{n-1} \delta(\varepsilon) \frac{c_{2}}{L^{\beta}}\left[\sum_{l \geq 2} \frac{1}{(l-1) !}\left(c_{3} \bar{g}\right)^{l-1}+\right. \\
& +k_{*}^{n-1} \delta(\varepsilon) \sum_{l \geq 2} \frac{1}{l !} \sum_{m=2}^{l}\left(\begin{array}{c}
l \\
m
\end{array}\right)\left(k_{*}^{n-1} \delta(\varepsilon)\right)^{m-2} \cdot \\
& \cdot\left(c_{3} \bar{g}^{l-m}\right] \cdot e^{\frac{\lambda_{*}}{2} L^{-\beta}|\varphi|^{2}} \\
& \leq k_{*}^{n-1} \delta(\varepsilon) \frac{c_{2}}{L^{\beta}} c_{3} \bar{g}\left[(1+0(\bar{g}))+k_{*}^{n-1} \delta(\varepsilon)(1+0(\bar{g}))\right] \cdot \\
& \cdot e^{\frac{\lambda_{*}}{2} L^{-\beta}|\varphi|^{2}}
\end{aligned}
$$


since the series converges. Hence,

$$
\left|\frac{d^{2}}{d \varphi} \Delta C_{n}\right| \leq k_{*}^{n-1} \delta(\varepsilon) \frac{c_{2} c_{3} \bar{g}}{L^{\beta}}\left(1+\varepsilon^{1 / 2}\right)^{2} e^{\frac{\lambda_{*}}{2} L^{-\beta}|\varphi|^{2}}
$$

similarly using part (ii) of Proposition 3 and $H_{n}$,

$$
\left|\Delta C_{n}\right| \leq k_{*}^{n-1} \delta(\varepsilon) c_{2} c_{3} \bar{g}\left(1+\varepsilon^{1 / 2}\right)^{2}\left[\frac{\varphi^{2}}{L^{\beta}} e^{\frac{\lambda_{*}}{2} L^{-\beta}|\varphi|^{2}}+1\right]
$$

Next we shall bound $\Delta D_{n}$ and its derivative. Starting from (B6), and using part (i) of Proposition 3, (5.21) and part (i) of $H_{n}$, (5.11), we get:

$$
\left|\frac{d}{d \varphi^{2}} \Delta D_{n}\right| \leq \frac{c_{2}}{L^{\beta}}\left[\sum_{l \geq 1} \frac{1}{l !} \sum_{1 \leq n \leq l}\left(\begin{array}{c}
l \\
m
\end{array}\right) \sum_{s=1}^{m}\left(\begin{array}{c}
m \\
s
\end{array}\right)\left(c^{(n-1)}\right)^{s}\left(c_{3} \bar{g}\right)^{l-m}\right] \cdot e^{\frac{\lambda_{*}}{2} L^{-\beta}|\varphi|^{2}}
$$

The series converges, and we deduce:

$$
\left|\frac{d}{d \varphi^{2}} \Delta D_{n}\right| \leq \frac{c_{2}}{L^{\beta}} \cdot c^{(n-1)} c e^{\frac{\lambda_{*}}{2} L^{-\beta}|\varphi|^{2}}
$$

In the same way, using part (ii) of Proposition 3 and part (i) of $H_{n}$ we get:

$$
\left|\Delta D_{n}\right| \leq c_{2} c^{(n-1)} c\left[\frac{\varphi^{2}}{L^{\beta}} e^{\frac{\lambda *}{2} L^{-\beta}|\varphi|^{2}}+1\right]
$$

whence,

$$
\left|\Delta D_{n}(0)\right| \leq c c_{2} c^{(n-1)}
$$

Now recall, (5.11 a) of $H_{n}$,

$$
c^{(n-1)}=\frac{\bar{g}}{L^{\bar{\beta} / 2}} k_{*}^{n-2} \delta(\varepsilon)+\frac{\bar{g}^{2}}{L^{\bar{\beta} / 2}}\left(\frac{1}{L^{\bar{\beta} / 2}}\right)^{n-1}
$$

Because of (5.6),

$$
\frac{1}{L^{\bar{\beta} / 2}}<\frac{1}{2} \epsilon^{5 / 2}=\frac{1}{2} \delta(\varepsilon)<k_{*} \delta(\varepsilon)
$$

Hence,

$$
c^{(n-1)} \leq k_{*}^{n-1} \delta(\varepsilon)\left[\frac{\bar{g}}{k_{*} L^{\bar{\beta} / 2}}+\frac{\bar{g}^{2}}{L^{\bar{\beta} / 2}}\right]
$$

Hence, from (B 13),

$$
\left|\Delta D_{n}(0)\right| \leq k_{*}^{n-1} \delta(\varepsilon)\left[\frac{c^{\prime} \bar{g}}{L^{\bar{\beta} / 2}}+\bar{g}^{2}\right]
$$

From the above bounds on $\Delta C_{n}$ and $\Delta D_{n}$ we get bounds on

$$
\Delta B_{n}=\Delta C_{n}+\Delta D_{n}
$$


From (B8) and (B14) we get

$$
\left|\Delta B_{n}(0)\right| \leq k_{*}^{n-1} \delta(\varepsilon)\left[1-\bar{g}\left(\frac{\lambda_{*}}{4 \pi}\right)^{d / 2}\left(1+\frac{1}{2} L^{-\beta}\right)+\frac{c^{\prime} \bar{g}}{L^{\bar{\beta} / 2}}+5 \varepsilon^{3 / 2}\right]
$$

This is (5.26). On the otherhand, from (B9) and (B11), we have,

$$
\begin{gathered}
\left|L^{\varepsilon} \frac{d}{d \varphi^{2}} \Delta B_{n}\right| \leq\left[\frac{c_{2} c_{3}^{\prime} \bar{g}}{L^{\bar{\beta}}} k_{*}^{n-1} \delta(\varepsilon)+\frac{c_{2} c}{L^{\bar{\beta}}} c^{(n-1)}\right] e^{\frac{\lambda_{*}}{2} L^{-\beta}|\varphi|} \\
\quad \leq \frac{c_{2} c^{\prime}}{L^{\bar{\beta} / 2}}\left[\frac{\bar{g}}{L^{\bar{\beta} / 2}} k_{*}^{n-1} \delta(\varepsilon)+\frac{1}{L^{\bar{\beta} / 2}} c^{(n-1)}\right] e^{\frac{\lambda_{*}}{2} L^{-\beta}|\varphi|}
\end{gathered}
$$

Now,

$$
\begin{aligned}
\frac{1}{L^{\bar{\beta}} / 2} c^{(n-1)} & =\frac{1}{L^{\bar{\beta} / 2}}\left(\frac{\bar{g}}{L^{\bar{\beta} / 2}} k_{*}^{n-2} \delta(\varepsilon)+\frac{\bar{g}^{2}}{L^{\bar{\beta} / 2}}\left(\frac{1}{L^{\bar{\beta} / 2}}\right)^{n-1}\right) \\
& =\frac{\bar{g}}{L^{\bar{\beta}}} k_{*}^{n-2} \delta(\varepsilon)+\frac{\bar{g}^{2}}{L^{\bar{\beta} / 2}}\left(\frac{1}{L^{\bar{\beta} / 2}}\right)^{n}
\end{aligned}
$$

Plugging this into the previous inequality,

$$
\begin{aligned}
\left|L^{\varepsilon} \frac{d}{d \varphi^{2}} \Delta B_{n}\right| & \leq \frac{c_{2} c^{\prime}}{L^{\bar{\beta}} / 2}\left[\frac{\bar{g}}{L^{\bar{\beta}} / 2} k_{*}^{n-1} \delta(\varepsilon)\left(1+\frac{1}{k_{*} L^{\bar{\beta} / 2}}\right)+\right. \\
+ & \left.\frac{\bar{g}^{2}}{L^{\bar{\beta} / 2}}\left(\frac{1}{L^{\bar{\beta} / 2}}\right)^{n}\right] \cdot e^{\frac{\lambda_{*}}{2} L^{-\beta}|\varphi|}
\end{aligned}
$$

We can now use the overall factors $L^{-\bar{\beta} / 2}$ to bound unecessary constants by $1 / 4$. We thus get:

$$
\left|L^{\varepsilon} \frac{d}{d \varphi^{2}} \Delta B_{n}\right| \leq \frac{1}{4}\left[\frac{\bar{g}}{L^{\bar{\beta} / 2}} k_{*}^{n-1} \delta(\varepsilon)+\frac{\bar{g}^{2}}{L^{\bar{\beta} / 2}}\left(\frac{1}{L^{\bar{\beta} / 2}}\right)^{n}\right] \cdot e^{\frac{\lambda *}{2} L^{-\beta}|\varphi|^{2}}
$$

which is (5.27). Finally from (B10) and (B12), we obtain similarly:

$$
\left|\Delta B_{n}\right| \leq c\left[\bar{g} k_{*}^{n-1} \delta(\varepsilon)\left(1+\frac{1}{k_{*} L^{\bar{\beta} / 2}}\right)^{n}+\bar{g}^{2}\left(\frac{1}{L^{\bar{\beta} / 2}}\right)^{n}\right] \cdot\left(\frac{\varphi^{2}}{L^{\beta}} e^{\frac{\lambda_{*}}{2} L^{-\beta}}|\varphi|^{2}+1\right)
$$

which is (5.28).

This completes the proof of Proposition 4. 


\section{REFERENCES}

1. D.R.Nelson, T.Piron and S.Weinberg (eds): Statistical Mechanics of Membranes and Surfaces, World Scientific, Singapore, 1989.

2. M.Kardar and D.R.Nelson: Phys. Rev. Lett 58 (1987) 1298, Phys. Rev. Lett 58 (1987) 2280, Phys. Rev. A 38 (1988) 966

3. J.A.Aronowitz and T.C.Lubensky, Europhys. Lett 4 (1987), 395

4. B.Duplantier, Phys. Rev. Lett 58 (1987) 2733

5. B.Duplantier, Phys. Rev. Lett 62 (1989) 2337

6. B.Duplantier, T.Hwa and M.Kardar, Phys. Rev. Lett 64, (1990) 2022.

7. F.David, B.Duplantier and E.Guitter: Phys. Rev. Lett. 70 (1993) 2205, Nucl. Phys. B 394 (1993), 555

8. J.Kogut and K.G.Wilson: Phys. Report 12 C (1974) 75

9. G.Gallavotti Memorie dell'Accademia dei Lincei XV, (1978) 23

10. G.Gallavotti Rev. of Modern Phys 57 (1985), 471

11. G.Gallavotti Lausanne Lectures (3 cycl.de la Suisse Romande 1990) D.C.Brydges: Lausanne Lectures ( $3^{\circ}$ cycl de la Suisse Romande 1992)

12. K.Osterwalder and R.Stora (eds), Critical Phenomena, Random Fields, Gauge Theoric Les Houches (1984), (North Holland, Amsterdam 1986)

13. K.Gawedzki and A.Kupiainen: Nucl. Phys. B 262, (1985) 33, Comm. math. Phys. 102, 1985, 1

14. K.Gawedzki and A.Kupiainen: Comm. Math, Phys, 89, 191 (1983) 